Pure and Applied Mathematics Quarterly

Volume 3, Number 1

(Special Issue: In honor of

Robert MacPherson, Part 3 of 3 )

$225-282,2007$

\title{
The Development of Intersection Homology Theory
}

\author{
Steven L. Kleiman
}

\section{Contents}

Foreword 225

1. Preface 226

2. Discovery 227

3. A fortuitous encounter 231

4. The Kazhdan-Lusztig conjecture 235

5. D-modules 238

6. Perverse sheaves 244

7. Purity and decomposition 248

8. Other work and open problems 254

References 260

9. Endnotes 265

References 279

\section{FOREWORD}

The first part of this history is reprinted with permission from "A century of mathematics in America, Part II," Hist. Math., 2, Amer. Math. Soc., 1989, pp. 543-585. Virtually no change has been made to the original text. However, the text has been supplemented by a series of endnotes, collected in the new

Received October 9, 2006. 
Section 9 and followed by a list of additional references. If a subject in the reprint is elaborated on in an endnote, then the subject is flagged in the margin by the number of the corresponding endnote, and the endnote includes in its heading, between parentheses, the page number or numbers on which the subject appears in the reprint below.

\section{Preface}

Intersection homology theory is a brilliant new tool: a theory of homology groups for a large class of singular spaces, which satisfies Poincaré duality and the Künneth formula and, if the spaces are (possibly singular) projective algebraic varieties, then also the two Lefschetz theorems. The theory was discovered in 1974 by Mark Goresky and Robert MacPherson. It was an unexpected find, but one highly suited to the study of singular spaces, and it has yielded profound results. Most notably, the Kazhdan-Lusztig conjecture was established via a remarkable bridge between representation theory and intersection homology theory provided by $\mathcal{D}$-module theory. In fact, in 1980 , the conjecture, which was a little over a year old, motivated the construction of that bridge, and the bridge in turn led to some far reaching new advances in intersection homology theory. All told, within a decade, the development of intersection homology theory had involved an unprecedented number of very bright and highly creative people. Their work is surely one of the grand mathematical endeavors of the century.

From a broader historical perspective, it is clear that the time was ripe for the discovery of intersection homology theory. Enormous advances had been made in the study of equisingular stratifications of singular spaces during the mid-to-late 1960s. During the early 1970s, various characteristic classes had been found for singular spaces, and there had been several investigations of Poincaré duality on singular spaces, although those investigations were concerned with the degree of failure of Poincaré duality rather than a modification of the homology theory.

In addition, about a year and a quarter after the discovery, while the new theory was still undeveloped and virtually unknown, Jeff Cheeger, pursuing an entirely different course of research from that of Goresky and MacPherson, independently discovered an equivalent cohomology theory for essentially the same class of singular spaces: a deRham-Hodge theory corresponding to their combinatorial theory. Furthermore, it is not surprising that there was, during the decade following the discovery of intersection homology theory, a great confluence of topology, algebraic geometry, the theory of differential equations, and representation theory. While those theories had diverged after Riemann, they had converged again on occasion in the hands of Poincaré around 1900, of Lefschetz around 1930, and of others in the 1950s and 1960s.

The present account of the frenetic development of intersection homology theory during the first decade or so after its discovery is intended simply to provide a 
feeling for who did what, when, where, how, and why, and a feeling for the many interpersonal lines of development. The mathematical discussions are not meant to be outlines or surveys; they are meant to be indications of the philosophy, the aims, the methods, and the material involved. The author has constantly striven to be impartial, historically and technically accurate, and reasonably thorough. Of course, here and there, a delicate line had to be drawn between what to include and what to leave out. The author regrets any errors and oversights.

The present account was based primarily on the rather lengthy literature. There are, first of all, several excellent survey articles [11], [94], [76], and [45].

Of course, it was still necessary to appeal to the original research papers to obtain a more complete and more rounded picture. Unfortunately, some of the historical remarks in print are inaccurate or misleading; their authors seem simply to have been unaware of the whole story.

The present account was also based on numerous interviews: brief interviews with M. Artin, J. Bernstein, R. Crew, D. Kazhdan, J.-L. Verdier, K. Vilonen, and D. Vogan; short interviews with A. Beilinson, J.-L. Brylinski, and S. Zucker; longer interviews with Cheeger, G. Lusztig, L. Saper; and an extended series of interviews with Goresky, D. T. Lê, and MacPherson. In addition, A. Altman, Beilinson, Brylinski, Cheeger, Goresky, B. Kleiman, Lê, Lusztig, J. Lützen, MacPherson, A. Thorup, Verdier, and Zucker read earlier versions of this account and made a number of suggestions, which led to significant improvements. Unfortunately, not everyone who was invited to comment did so. However, it is a pleasure now to thank each and every one of those who did contribute for their invaluable help; the article is all the better for it.

\section{Discovery}

Intersection homology theory was discovered during the fall of 1974 at the IHES (Institut des Hautes Études Scientifiques) in Paris by Mark Goresky and Robert MacPherson. They were seeking a theory of characteristic numbers for complex analytic varieties and other singular spaces. During the preceding four years, the Whitney classes of Dennis Sullivan, the Chern classes of MacPherson, and the Todd classes of Paul Baum, William Fulton and MacPherson had been discovered for such spaces. (The existence of the Chern classes had been conjectured in 1970 by Alexandre Grothendieck and Pierre Deligne, and then the classes had been constructed by MacPherson in a manuscript of July 25, 1972. In 1978, Jean-Paul Brasselet and Marie-Hélène Schwartz proved that the classes correspond under Alexander duality to the cohomology classes that Schwartz had introduced in 1965. The classes are often called the Chern classes of Schwartz and MacPherson.) 
All those classes are homology classes, however, and homology classes cannot be multiplied. So Goresky and MacPherson figured, in analogy with the secondary homology operations, that there would be certain "intersectable" homology classes, whose intersection product would be unambiguous modulo certain "indeterminacy" classes.

Goresky was, at the time, writing his Ph.D. thesis under MacPherson's direction on a geometric treatment of cohomology groups, viewing them as the homology groups of a certain kind of cycle. (The thesis was submitted to Brown University in June 1976 and published as [36] in 1981.) By then, they knew why two "geometric cocycles" on a complex analytic variety $X$ can be intersected: one can be made transverse to the other and to each stratum $S_{\alpha}$ in a Whitney stratification of $X$.

A Whitney stratification is a particularly nice (locally finite) partition of a complex analytic variety $X$ into disjoint locally closed, smooth analytic strata $S_{\alpha}$. It satisfies the following 'boundary condition': each closure $\bar{S}_{\alpha}$ is a union of strata $S_{\beta}$. Also, Whitney's condition (B) holds: if a sequence of points $a_{i} \in S_{\alpha}$ and a sequence of points $b_{i} \in S_{\beta}$ both approach the same point $b \in S_{\beta}$, then the limit of the secant lines connecting $a_{i}$ to $b_{i}$ lies in the limit of the tangent spaces to $S_{\alpha}$ at $a_{i}$ if both limits exist. Consequently, the Thom-Mather isotopy theorem obtains: the stratification is locally topologically trivial along $S_{\beta}$ at $b$. Whitney (1965) proved that given any (locally finite) family of locally closed, smooth analytic subvarieties $Y_{i}$ of $X$, whose closures are analytic, there exists a Whitney stratification such that each $Y_{i}$ is a union of strata.

Goresky and MacPherson found a suitable more general setup in which to intersect geometric cocycles: it is sufficient that $X$ be a piecewise linear space, or pl-space, with a stratification defined by closed subsets

$$
X=X_{n} \supset X_{n-1} \supset X_{n-2} \supset X_{n-3} \supset \cdots \supset X_{1} \supset X_{0}
$$

such that

(1) $X_{n-1}=X_{n-2}$;

(2) each stratum $X_{i}-X_{i-1}$ is empty or is a pl-manifold of (pure) topological dimension $i$ along which the normal structure of $X$ is locally trivial (more precisely, each point $x$ of the stratum $X_{i}-X_{i-1}$ admits a closed neighborhood $U$, in $X$, pl-homeomorphic to $B^{i} \times V$, where $B^{i}$ is the closed ball of dimension $i$ and $V$ is a compact space with a filtration by closed subsets,

$$
V=V_{n} \supset V_{n-1} \supset \cdots \supset V_{i}=\mathrm{pt}
$$

and the homeomorphism preserves the filtration; that is, it carries $U \cap X_{j}$ onto $\left.B^{i} \times V_{j}\right)$

(3) the closure of each stratum is a union of strata;

(4) the largest stratum $X_{n}-X_{n-2}$ is oriented and dense. 
Goresky and MacPherson made several attempts to relax the transversality condition on the cycles by allowing a (piecewise linear and locally finite) $i$-cycle to deviate from dimensional transversality to $X_{n-k}$ within a tolerance specified by a function $\bar{p}(k)$, which is independent of $i$; that is, the $i$-cycle is allowed, for each $k$, to intersect $X_{n-k}$ in a set of dimension as much as $i-k+\bar{p}(k)$. They called the function $\bar{p}(k)$ the perversity. It is required to satisfy these conditions:

$$
\bar{p}(2)=0 \quad \text { and } \quad \bar{p}(k+1)= \begin{cases}p(k), & \text { or } \\ p(k)+1 . & \end{cases}
$$

The first condition guarantees that the $i$-cycle lies mostly in the nonsingular part of $X$, where it is orientable. The second condition says that the perversity function is nondecreasing and grows no faster than by 1 . That condition was not imposed until the summer of 1975 (see below).

All of a sudden one day, Goresky and MacPherson realized that the cycles should be identified by homologies that were allowed to deviate in the same way. Thus they obtained a spectrum of new groups. They called them the "perverse homology" groups, and used that name for about six months. Then Sullivan convinced them to change it, suggesting "Schubert homology" and "intersection homology." The rest is history!

The intersection homology groups $I H_{i}^{\bar{p}}(X)$ are finitely generated when $X$ is compact. When $X$ is normal, the groups range from the ordinary cohomology groups, where $\bar{p}(k)=0$ for all $k$, to the ordinary homology groups, where $\bar{p}(k)=$ $k-2$ for all $k$. In addition, the groups possess intersection pairings

$$
I H_{i}^{\bar{p}}(X) \times I H_{j}^{\bar{q}}(X) \longrightarrow I H_{i+j-n}^{\bar{p}+\bar{q}}(X)
$$

generalizing the usual cup and cap products.

Goresky and MacPherson filled a whole notebook with examples. They felt sure that they were on to something. However, to their dismay, the theory appeared to be tied tightly to the stratification and rather artificial. Then, to see if perchance they had come any further along toward a theory of characteristic numbers, they decided to focus on one characteristic number, the signature. Indeed, in 1970, Sullivan had posed the problem of finding a class of singular spaces with a cobordism invariant signature. The key ingredient here, of course, is Poincaré duality.

Suddenly, they realized that, just as cohomology groups and homology groups are dually paired, so too the intersection homology groups of complementary dimension $(i+j=n)$ and complementary perversity $(\bar{p}(k)+\bar{q}(k)=k-2)$ should be dually paired. They opened the notebook and were astonished to find that the ranks of the complementary groups were indeed always the same. In fact, there was one example where the ranks appeared at first to be different, but they soon located an error in the calculations. There was no doubt about 
it: Poincaré duality must hold! In particular, Sullivan's problem was clearly solved: if $X$ is compact, of dimension $4 l$, and analytic or simply has only even codimensional strata, then the middle perversity group $I H_{2 l}^{\bar{m}}(X)$, where $\bar{m}(k)=$ $\left\lfloor\frac{k-2}{2}\right\rfloor$, must carry a nondegenerate bilinear form, whose signature is invariant under cobordisms with even codimensional strata (but not under homotopy). It was a magic moment!

After a week or two of very intense effort, Goresky and MacPherson had the essence of the first proof of duality. It was geometric, technical, and messy: they used the Leray spectral sequence of the link fibration over each stratum and then the Mayer-Vietoris sequence to patch. They went to Sullivan and John Morgan, who were also at the IHES, and told them about their discovery. Sullivan for once was dumbfounded. Morgan probably said, "Come on, you can't fool around with the definition of homology." However, Morgan quickly saw the point, and used the new ideas to finish Sullivan's program of giving a geometric proof of Poincaré's Hauptvermutung [63, p. 1176] unfortunately, the proof is technical and complicated and has not yet been put in print.

Years passed before Goresky and MacPherson succeeded in writing up and publishing their work. They had not even wanted to start until they had analyzed the invariance of the groups $I H_{i}^{\bar{p}}(X)$ under restratification, and it was not until the summer of 1975 that that they discovered that the growth condition $\bar{p}(k) \leq \bar{p}(k+1) \leq \bar{p}(k)+1$ implies that invariance. Moreover, they did not know what category to work in: differentiable, pl, or topological. When they finally settled on the pl-category, they realized that pl-transversality should say that two pl-chains can be made transverse within each stratum. Clint McCrory, an expert on pl-topology, was at Brown University with them during the academic year 1975-1976, and they asked him about the transversality. He immediately gave them a proof and published it so that they could refer to it.

During the summer of 1976, Goresky and MacPherson struggled with another technical problem. They needed a single chain complex with which to define the intersection homology groups, they needed to be able to move two chains into transverse relative position to intersect them, and they needed to find a dual complex with the same properties. The problem was that all those properties seemed to be technically incompatible. They finally discovered that they had to take the chain complex that is the direct limit over all triangulations to get enough flexibility. They also discovered certain sets $Q(i, p)$ and $L(i, p)$, which are like "perverse skeletons" of the spaces and which allowed them to to prove Poincaré duality without the Leray spectral sequence with coefficients in the intersection homology groups of the fiber in the link fibration.

In addition, Goresky and MacPherson had other serious mathematical projects in progress during those years. Goresky had to write up his thesis. MacPherson was working with Fulton on a literally revolutionary new approach to intersection 
theory in algebraic geometry, [32], [33]. Some other projects involved exciting new ideas in intersection homology theory, which completely captured their attention for months at a time; those ideas will be discussed below.

When Izrail Gelfand visited Paris in the fall of 1976, he met MacPherson and convinced him to write up and publish an announcement of the discovery of intersection homology theory; it [39] appeared in the spring of 1977. With that in print, Goresky and MacPherson felt less pressure to drop everything else, and they did not get back to writing up the detailed treatment until the summer of 1978. Then they worked very hard on the exposition, and, in September 1978, they submitted it for publication. It took almost a year to be refereed and did not appear until 1980 as [40].

\section{A fortuitous encounter}

At a Halloween party near Paris in 1976, Deligne asked MacPherson what he was working on and was told about intersection homology theory. At the time, Deligne was thinking about the Weil conjectures, monodromy, and the hard Lefschetz theorem. He was also thinking about Steven Zucker's work in progress on the variation of Hodge structures over a curve (which eventually appeared in [98]), wondering in particular about how to extend it to higher dimensions. Earlier, Deligne had made significant contributions to the theory of duality of quasi-coherent sheaves (March 1966) and to the formulation and solution of a generalized Riemann-Hilbert problem (fall of 1969). Thus Deligne had been led to the idea of truncating the pushforth of a local system, or locally constant sheaf of vector spaces, on the complement of a divisor with normal crossings on a smooth complex ambient variety $X$ of topological dimension $n=2 d$.

The party was at one of the IHES's large residences, and almost everyone from the institute was there. On a scrap of paper, Deligne wrote down for the first time his celebrated formula,

$$
I H_{i}^{\bar{p}}(X)=H^{2 d-i}\left(\mathbf{I C}_{\bar{p}}(X)\right),
$$

expressing the intersection homology groups of $X$, equipped with a suitable stratification by closed sets $\left\{X_{i}\right\}$, as the hypercohomology of the following complex of sheaves:

$$
\mathbf{I C}_{\bar{p}}(X):=\tau_{\leq \bar{p}(2 d)} \mathbf{R} i_{2 d *} \cdots \tau_{\leq \bar{p}(2)} \mathbf{R} i_{2 *} \mathbb{C}_{X-X_{2 d-2}}
$$

where $\mathbb{C}_{X-X_{2 d-2}}$ is the complex consisting of the constant sheaf of complex numbers concentrated in degree 0 , where $i_{k}$ is the inclusion of $X-X_{2 d-k}$ into $X-X_{2 d-k+1}$, and where $\tau_{\leq k}$ is the truncation functor that kills the stalk cohomology in degree above $k$. The complex $\mathbf{I C}_{\bar{p}}(X)$ is, however, well defined only in the 'derived category' - the category constructed out of the category of complexes up to homotopy equivalence, by requiring a map of complexes to be an 
isomorphism (to possess an inverse) if and only if it induces an isomorphism on the cohomology sheaves.

Deligne asked about a key example, the local intersection homology groups at an isolated singularity. MacPherson responded immediately: they are the homology groups of the link (the retract of a punctured neighborhood) in the bottom half dimensions and 0 in the middle and in the top half dimensions. That answer was exactly what Deligne obtained from his construction. They conjectured that the formula is correct.

Deligne, it seems, had always worked before with a smooth ambient variety and with twisted coefficients. He was rather surprised to learn from MacPherson that there might be a significant theory on a singular space. He could see, however, that his construction would yield cohomology groups that satisfy Poincaré duality because of the Verdier-Borel-Moore duality in the derived category of complexes of sheaves. MacPherson, on the other hand, was surprised at the entrance of the derived category. However, he could see that Deligne's construction might have great technical advantages.

At the time, MacPherson was in the midst of giving a series of lectures on intersection homology theory, and Jean-Louis Verdier was in the audience. Verdier expressed considerable interest in the theory and in Deligne's formula. During the ensuing weeks, he explained more about the derived category and duality to MacPherson.

The next academic year, 1977-1978, MacPherson was back at Brown, and Goresky was in his second and final year as a Moore Instructor at MIT. MacPherson showed Goresky the scrap of paper with Deligne's formula on it and said: "We have to learn derived categories to understand this formula!" In a seminar on intersection homology theory at Brown, they worked out a proof of the formula. The proof was long and messy, involving the derived category of simplicial sheaves and a limit over simplicial subdivisions.

During the following academic year, 1978-1979, Goresky and MacPherson wrote up that proof as part of a first draft of their paper [42], which doubtless is the single most important paper on topological intersection homology theory. However, they were unhappy with that complicated first treatment and decided to streamline it. They made steady progress during the next year, 1979-1980. They found several axiomatic characterizations of $\mathbf{I C}_{\bar{p}}(X)$ among all complexes in the derived category whose cohomology sheaves are constructible with respect to the given stratification. (A sheaf of $\mathbb{Q}$-vector spaces is called constructible with respect to a stratification by closed sets $\left\{X_{i}\right\}$ if its stalks are finite dimensional and its restriction to each stratum $X_{i}-X_{i-1}$ is locally constant.) They found that the "constructible derived category' is a "paradise," as Verdier called it: it possesses some two dozen natural properties. However, progress was hampered 
because Goresky was in Vancouver and MacPherson was in Providence during those years.

The first copy of [42] that was submitted for publication was lost in the mail from Vancouver, and that horrible fact was not discovered for six or eight months. The paper was immediately resubmitted in June 1981. Meanwhile, many people had read the manuscript and offered pages of corrections and suggestions. Their comments were incorporated in a major revision of the paper, which was resubmitted in December 1982. Finally, the paper appeared in print in early 1983, nearly six and a half years after the Halloween party.

In $\S 1$ of the paper, Goresky and MacPherson develop the general theory of the constructible derived category. In $\S 2$, they study pl-pseudomanifolds $X$, and show how the construction of the $I H_{i}^{\bar{p}}(X)$ in their first paper [40] actually yields a complex of sheaves. In $\S 3$, they develop a first axiomatic characterization $\mathbf{I C}_{\overline{\bar{p}}}(X)$, and use it to prove Deligne's formula. In $\S 4$ of the paper, Goresky and MacPherson give a second axiomatic characterization of $\mathbf{I C}_{\bar{p}}(X)$, which they derive from the first. It does not involve the stratification, and yields the following remarkable theorem.

Theorem $[42,4.1]$. The intersection homology groups $I H_{i}^{\bar{p}}(X)$ are topological invariants; in fact, for any homeomorphism $f: X \rightarrow Y$, the complexes $\mathbf{I C}_{\overline{\bar{p}}}(X)$ and $f^{*} \mathbf{I C}_{\bar{p}}(Y)$ are isomorphic in the derived category.

Earlier, in the summer of 1975, Goresky and MacPherson had figured out that the groups $I H_{i}^{\bar{p}}(X)$ are independent of the stratification, but they still needed a pl-structure. So, in 1976, Goresky spent some time working with singular chains, but he bumped into an obstacle. About nine years later, Henry King [59] independently worked out a theory based on singular chains and, without using sheaf theory, he recovered the topological invariance.

In $\S 5$, Goresky and MacPherson reproved using sheaf theory some of the basic properties of the intersection homology groups, such as the existence of the intersection pairing and the validity of Poincaré duality. They also proved some new results, such as the following comparison theorem.

Theorem (Comparison) [42, 5.6.3]. If $X$ is a complex algebraic variety that is compact, normal, and a local complete intersection, and if $\bar{p}(k) \geq k / 2$ for $k \geq 4$, then $I H_{i}^{\bar{p}}(X)=H_{i}(X)$ for all $i$.

In $\S 6$, Goresky and MacPherson proved several theorems about complex algebraic varieties $X$ of (algebraic) dimension $d$ and the middle perversity $\bar{m}(k)=$ $\left\lfloor\frac{k-2}{2}\right\rfloor$. This case is particularly important. So, to lighten the notation, set

$$
\text { IC }(X):=\mathbf{I C}_{\bar{m}}(X), \quad I H_{i}(X):=I H_{i}^{\bar{m}}(X), \quad \text { and } \quad I H^{i}(X):=I H_{2 d-i}(X) .
$$

The first theorem of $\S 6$ gives a third and the most important version of the axiomatic characterization of $\mathbf{I C} \cdot(X)$. 
Theorem [42, 6.1]. Consider the derived category of bounded complexes $\mathbf{K}$ of sheaves such that the cohomology sheaves $\mathbf{H}^{i}(\mathbf{K})$ are constructible with respect to some Whitney stratification, which depends on $\mathbf{K}$. Then, in this category, there is a unique complex $\mathbf{K}$ satisfying the following conditions:

(a) (Normalization) There is a dense open subset $U$ such that $\mathbf{H}^{i}(\mathbf{K}) \mid U=0$ for $i \neq 0$ and $\mathbf{H}^{0}(\mathbf{K}) \mid U=\mathbb{C}_{U}$.

(b) (Lower bound) $\mathbf{H}^{i}(\mathbf{K})=0$ for all $i<0$.

(c) (Support) $\operatorname{codim}\left(\operatorname{Supp}\left(\mathbf{H}^{i}(\mathbf{K})\right)\right)>i$ for all $i>0$.

(d) (Duality) $\mathbf{K}$ is isomorphic to its Verdier-Borel-Moore dual $\mathbf{K}^{\llcorner}$.

Condition (d) may be replaced by the following dual condition:

$\left(\mathrm{d}^{\ulcorner}\right)$(Cosupport) $\operatorname{codim}\left(\operatorname{Supp}\left(\mathbf{H}^{i}\left(\mathbf{K}^{\ulcorner}\right)\right)\right)>i$ for all $i>0$.

Moreover, $\mathbf{K}=\mathbf{I C} \cdot(X)$.

Goresky and MacPherson used this characterization to prove the following two theorems.

Theorem (Small resolution) [42, 6.2]. If a proper algebraic map $f: X \rightarrow Y$ is a small resolution, that is, if $X$ is smooth and for all $r>0$,

$$
\operatorname{codim}\left\{y \in Y \mid \operatorname{dim} f^{-1}(y) \geq r\right\}>2 r,
$$

then $I H_{i}(Y)=I H_{i}(X)=H_{i}(X)$; in fact, $\mathbf{R} f_{*} \mathbb{C}_{X}=\mathbf{I C} \cdot(Y)$.

Theorem (Künneth formula) [42, 6.3]. If $X$ and $Y$ are varieties, then

$$
I H_{i}(X \times Y)=\bigoplus_{j+k=i} I H_{j}(X) \otimes I H_{k}(X) .
$$

The Künneth formula had already been proved analytically by Jeff Cheeger. Although Goresky and MacPherson referred to Cheeger's article [21] for that proof, the proof did not actually appear explicitly in print before the article's sequel $[22, \S 7.3]$. For the unusual story of Cheeger's work, see the beginning of $\S 8$.

Later in $[45, \S \mathrm{A}]$, Goresky and MacPherson gave two interesting examples concerning small resolutions. In each example, there is a variety $Y$ with two different small resolutions $f_{1}: X_{1} \rightarrow Y$ and $f_{2}: X_{2} \rightarrow Y$ such that the induced vector space isomorphism between the cohomology rings of $X_{1}$ and $X_{2}$ is not a ring isomorphism.

In $[42, \S 7]$, Goresky and MacPherson gave a sheaf-theoretic proof of the following theorem, known as the 'Lefschetz hyperplane theorem' or the 'weak Lefschetz theorem'.

Theorem (Lefschetz hyperplane) [42, 7.1]. If $X$ is a projective variety of (algebraic) dimension $d$ and if $H$ is a general hyperplane, then for all $i$ the inclusion $\alpha: X \cap H \rightarrow X$ induces a map

$$
\alpha_{*}: I H_{i}(X \cap H) \longrightarrow I H_{i}(X) .
$$


Moreover, $\alpha_{*}$ is bijective for $i<d-1$ and surjective for $i=d-1$.

In fact, the theorem is proved not only for the middle perversity $\bar{m}$, but also for any perversity $\bar{p}$ such that $\bar{p}(k) \leq k / 2$. Hence, the theorem has the following corollary, whose second assertion results from the comparison theorem stated above.

Corollary [42, 7.4.1, 7.4.2]. If $X$ is normal, then the Gysin map of ordinary cohomology theory $\alpha^{*}: H^{i}(X \cap H) \rightarrow H^{i}(X)$ is bijective for $i>d-1$ and surjective for $i=d-1$. If $X$ is a normal local complete intersection, then the induced map on the ordinary homology groups $\alpha_{*}: H_{i}(X \cap H) \rightarrow H_{i}(X)$ is bijective for $i<d-1$ and surjective for $i=d-1$.

The sheaf-theoretic proof of the Lefschetz hyperplane theorem is like that in $[90$, XIV 3]. In $[42, \S 7]$ and in several other places in the literature of intersection homology theory, the latter proof is attributed to Michael Artin. However, Artin says that it is inappropriate to credit the proof to him, because the entire seminar, [90], is a report on joint work and, moreover, that particular proof is due to Grothendieck.

Goresky and MacPherson had learned from Deligne that the sheaf-theoretic proof of the Lefschetz theorem in [90, XIV 3] would carry over to intersection homology theory, and they presented the details in [42, §7]. However, they had already considered the theorem from two other points of view. First, in the summer of 1977, Cheeger and MacPherson had met and conjectured that the related 'hard Lefschetz theorem' and all the other various consequences of Hodge theory should hold for intersection homology theory; for more information about the conjecture, see the beginning of $\S 8$. Second, during 1978-1979, Goresky and MacPherson began work on their new stratified Morse theory. That winter, they found they could adapt Thom's Morse-theoretic argument in the nonsingular case to prove the Lefschetz theorem in the singular case. They gave that proof in $[43,5.4]$. (René Thom gave his proof in a lecture at Princeton in 1957. It was entered into the public domain in 1959 independently by Raul Bott and by Aldo Andreotti and Theodore Frankel.)

\section{The Kazhdan-Lusztig COnJeCture}

The Kazhdan-Lusztig conjecture grew out of a year of collaboration in Boston starting in the spring of 1978 between David Kazhdan and George Lusztig. Two years earlier, Tony Springer had introduced an important new representation on $l$-adic étale cohomology groups, of the Weyl group $W$ of a semisimple algebraic group over a finite field. Kazhdan and Lusztig found a new construction of the representation. Moreover, they allowed the ground field to be $\mathbb{C}$ as well. Indeed, they preferred $\mathbb{C}$ and the classical topology. Their work eventually appeared in their paper [58]. 
The representation module has two natural bases, and Kazhdan and Lusztig tried to identify the transition matrix. Thus they were led to define some new polynomials $P_{y, w}$ with integer coefficients indexed by the pairs of elements $y, w$ of $W$ with $y \leq w$, for any Coxeter group $W$.

Those two bases reminded Kazhdan and Lusztig of the two natural bases of the Grothendieck group of infinite dimensional representations of a complex semisimple Lie algebra g: the basis formed by the Verma modules $M_{\lambda}$ and that by the simple modules $L_{\mu}$. (By definition, $M_{\lambda}$ is the maximal irreducible module with highest weight $\lambda$, and $L_{\lambda}$ is its unique simple quotient.) Putting aside their work on the Springer resolution, Kazhdan and Lusztig focused on the transition matrix between the $M_{\lambda}$ and $L_{\mu}$. Work by Jens Carsten Jantzen and by Anthony Joseph along with some well-known examples, which indicated that the transition matrix might depend on the topology of the Schubert varieties $X_{w}$, the closures of the Bruhat cells $B_{w}$, led Kazhdan and Lusztig to formulate the following conjecture. The particular formulation below was taken from Lusztig's paper [72], but the original conjecture appeared in their joint paper [56], which was received for publication on March 11, 1979.

Conjecture (Kazhdan-Lusztig) [56, 1.5], [72, (4.4), (4.5)]. In the Grothendieck group,

$$
\begin{aligned}
L_{-\rho w-\rho} & =\sum_{y \leq w}(-1)^{l(w)-l(y)} P_{y, w}(1) M_{-\rho y-\rho} \\
M_{\rho w-\rho} & =\sum_{w \leq y} P_{w, y}(1) L_{\rho y-\rho}
\end{aligned}
$$

where, as usual, $\rho$ is half the sum of the positive roots, and $l(w):=\operatorname{dim}\left(X_{w}\right)$.

Kazhdan and Lusztig defined the polynomials $P_{y, w}$ by an effective combinatorial procedure, but it is poorly suited for actual computation. However, for restricted Weyl groups of type $A_{N}$, Alain Lascoux and Marcel Schützenberger [65] found that the polynomials satisfy some simpler recursion relations determined by the combinatorics, and, using a computer, they worked out some examples. Sergei Gelfand (Izrail Gelfand's son) and MacPherson [35, §5] discussed the KazhdanLusztig algorithm and worked out some examples by hand. Goresky [38], inspired by the latter treatment, implemented the algorithm on a VAX 11 and worked out the cases $A_{3}, A_{4}, A_{5}, B_{3}=C_{3}, B_{4}=C_{4}, D_{4}$, and $H_{3}$; the case of $A_{5}$ alone took 3 hours of CPU time. In addition, according to Lusztig, Dean Alvis implemented the cases of $E_{6}$ and $H_{4}$, but the results are too lengthy to print out in full. The study of the polynomials is rather important and has continued. According to MacPherson, recently (1988) Brian Boe, Thomas Enright, and Brad Shelton have generalized the work of Lascoux and Schützenberger to some other types of Weyl groups, and Kazhdan has made the interesting conjecture that $P_{y, w}$ depends only on the partially ordered set of $z$ between $y$ and $w$. 
Kazhdan and Lusztig said [56, top of p. 168] that " $P_{y, w}$ can be regarded as a measure for the failure of local Poincaré duality" on the Schubert variety $X_{w}$ in a neighborhood of a point of the Bruhat cell $B_{y}$. In the appendix, they discussed "some algebraic geometry related to the polynomials," but there they worked exclusively over the algebraic closure of a finite field of characteristic $p$, and used étale cohomology groups with coefficients in the $l$-adic numbers $\mathbb{Q}_{l}$ with $l \neq p$.

Kazhdan and Lusztig asked Bott about Poincaré duality on a singular space, and Bott sent them to MacPherson. Actually, Lusztig had already learned about intersection homology theory the year before in the spring of 1977 at the University of Warwick, England. At the time, he was on the faculty there. MacPherson came to Warwick and gave a lecture on the theory; after the talk, they discussed it further. Now, Kazhdan, Lusztig, and MacPherson had several discussions in person and by mail. Kazhdan and Lusztig were taken by all the ideas, and at MacPherson's suggestion, they wrote to Deligne. Deligne responded from Paris on April 20, 1979, with a seven-page letter. That letter has often been photocopied and often been cited, because it is the first tangible place where Deligne discussed his sheaf-theoretic approach.

In his letter, Deligne observed that the sheaf-theoretic approach works equally well for a projective variety $X$ over the algebraic closure of a finite field of characteristic $p$ with the étale topology and sheaves of $\mathbb{Q}_{l}$-vector spaces, $l \neq p$. The strata must be smooth and equidimensional, but it is unnecessary that the normal structure of $X$ be locally trivial in any particular sense along each stratum; it suffices that the stratification be fine enough so that all the sheaves involved are locally constant on each stratum. (In positive characteristic, a Whitney stratification need not exist, and if there is no special hypothesis on the normal structure, then the sheaves $\mathbf{H}^{i}(\mathbf{I C} \cdot(X))$ need no longer be constructible with respect to a given stratification; nevertheless, the sheaves will be constructible with respect to some finer stratification.)

Deligne stated that Poincaré duality and the Lefschetz fixed-point formula are valid. The latter applies notably to the Frobenius endomorphism $\phi_{q}: X \rightarrow X$, which raises the coordinates of a point to the $q$-th power, and which is defined when $q:=p^{e}$ is large enough so that the coefficients of a set of equations defining $X$ lie in the field $\mathbf{F}_{q}$ with $q$ elements. The fixed-points $x$ of $\phi_{q}$ are simply the points $x \in X$ with coordinates in $\mathbf{F}_{q}$, and the formula expresses their number as the alternating sum of the traces of $\phi_{q}$ on the $I H^{i}(X)$.

Deligne said, however, that he could not prove the following statement of purity: for every fixed-point $x$ and for every $i$, the eigenvalues of $\phi_{q}$ on the stalk at $x$ of the sheaf $\mathbf{H}^{i}(\mathbf{I C} \cdot(X))$ are algebraic numbers whose complex conjugates all have absolute value at most $q^{i / 2}$. Deligne said that he lacked enough evidence to call the statement a "conjecture," but he did call it a "problem." The problem was solved about fourteen months later by Ofer Gabber, see the beginning of $\S 7$. 
Deligne noted that if 'purity' holds, then so will the following two theorems, which Kazhdan and Lusztig had asked about. (In the statement of the second theorem, it is implicitly assumed that an isomorphism $\mathbb{Q}_{l}(1) \cong \mathbb{Q}_{l}$ has been fixed.) Indeed, given 'purity', then the methods and results of Deligne's second great paper on the Weil conjectures, [29], which was nearly finished at the time, will yield these theorems.

Theorem (Weil-E. Artin-Riemann hypothesis). For every $i$, the eigenvalues of the Frobenius map $\phi_{q}$ on $I H^{i}(X)$ are algebraic numbers whose complex conjugates are all of absolute value $q^{i / 2}$.

Theorem (Hard Lefschetz). If $[H] \in H^{2}\left(\mathbf{P}^{N}\right)$ denotes the fundamental class of a hyperplane $H$ in the ambient projective space, then for all $i$, intersecting $i$ times yields an isomorphism,

$$
(\cap[H])^{i}: I H^{d-i}(X) \stackrel{\sim}{\longrightarrow} I H^{d+i}(X) \quad \text { where } d:=\operatorname{dim}(X) .
$$

Kazhdan and Lusztig then solved the problem of purity directly in case of the Schubert varieties $X_{w}$ by exploiting the geometry. In fact, they proved the following stronger theorem.

Theorem [57, 4.2]. The sheaf $\mathbf{H}^{2 j+1} \mathbf{I C} \cdot\left(X_{w}\right)$ is zero. On the stalk at a fixed point, $\mathbf{H}^{2 j} \mathbf{I C} \cdot(X)_{x}$, the eigenvalues of $\phi_{q}$ are algebraic numbers whose complex conjugates all have absolute value exactly $q^{j}$.

On the basis of those theorems, Kazhdan and Lusztig then proved their main theorem.

Theorem [57, 4.3]. The coefficients of $P_{y, w}$ are positive. In fact,

$$
\sum_{j} \operatorname{dim}\left(\mathbf{H}^{2 j} \mathbf{I} \mathbf{C} \cdot\left(X_{w}\right)_{y}\right) q^{j}=P_{y, w}(q),
$$

where the subscript ' $y$ ' indicates the stalk at the base point of the Bruhat cell $B_{y}$.

\section{D-MOdUles}

By good fortune, the theory that was needed to establish the Kazhdan-Lusztig conjecture was actively being developed at the very same time as the work in intersection homology theory and representation theory, although quite independently. That theory was needed as much for its spirit as for its results. The theory is a sophisticated modern theory of linear partial differential equations on a smooth complex algebraic variety $X$ (see for example [5], [6], [68]). It is sometimes called microlocal analysis, because it involves analysis on the cotangent bundle $T^{*} X$ (although the term 'microlocal analysis' is also used more broadly to include more traditional topics in analysis on $\left.T^{*} X\right)$. It is sometimes called $\mathcal{D}$-module theory, because it involves sheaves of modules $\mathcal{M}$ over the sheaf of rings of holomorphic linear partial differential operators of finite order $\mathcal{D}:=\mathcal{D}_{X}$; these 
rings are noncommutative, left and right Noetherian, and have finite global homological dimension. It is sometimes called algebraic analysis because it involves such algebraic constructions as $\operatorname{Ext}_{\mathcal{D}}^{i}(\mathcal{M}, \mathcal{N})$. The theory as it is known today grew out of the work done in the 1960s by the school of Mikio Sato in Japan.

During the 1970s, one of the central themes in $\mathcal{D}$-module theory was David Hilbert's twenty-first problem, now called the Riemann-Hilbert problem. "This problem," Hilbert [49] wrote, "is as follows: To show that there always exists a linear differential equation of Fuchsian class with given singular points and monodromic group." It is "an important problem, one which very likely Riemann himself may have had in mind." Here Hilbert was, doubtless, thinking of Riemann's 1857 paper on Gauss's hypergeometric equation and of Riemann's 1857 related unfinished manuscript, which was published posthumously in his collected works in 1876 .

The hypergeometric equation is of order 2 and has singular points at 0,1 , and $\infty$, but in the manuscript Riemann began a study of $n$th order equations with $m$ singular points. Riemann's ingenious idea was to obtain information about the equations and the solutions from the monodromy groups (each group consists of the linear transformations undergone by a basis of solutions as they are analytically continued along closed paths around a singular point). He assumed at the outset that, at a singular point $x$, each solution has the form

$$
(z-x)^{s}\left[\phi_{0}+\phi_{1} \log (z-x)+\cdots+\phi_{\lambda} \log ^{\lambda}(z-x)\right]
$$

where $s$ is some complex number and the $\phi$ 's are meromorphic functions.

Guided by Riemann's paper, Lazarus Fuchs and his students in 1865 took up the study of $n$th order equations (see [63, p. 724]),

$$
y^{(n)}+a_{1}(z) y^{(n-1)}+\cdots+a_{n}(z) y=0 .
$$

Fuchs showed that for the solutions to have the form described above it is necessary and sufficient that $(z-x)^{i} a_{i}(z)$ be holomorphic at $x$ for all $i$ and $x$. An equation whose coefficients $a_{i}(z)$ satisfy this condition is said to have regular singular points or to be regular, although Fuchs used a different term. Fuchs gave special consideration to the class of equations that have at worst regular singular points in the extended complex plane, and so such equations are said to be of Fuchsian class or type.

The original Riemann-Hilbert problem was given its first complete solution in 1905 by Hilbert himself and by Oliver Kellogg using the theory of integral equations (see [63, ], p.726]) and in 1913 by George David Birkhoff using a method of successive approximations. Birkhoff added the concepts of a canonical system of differential equations and the equivalence of such systems (and he attacked the case of irregular singular points). The concept of a canonical system is not now present in $\mathcal{D}$-module theory, but, according to Lê Dũng Tráng, it would be good to introduce one and develop it appropriately. 
In the fall of 1969, Deligne [27] made a particularly significant advance: he generalized the problem greatly and solved it as follows. Given an open subset $U$ of a smooth complex algebraic variety $X$ of arbitrary dimension $d$ such that the complement $X-U$ is a divisor with normal crossings (that is, locally it is analytically isomorphic to the union of coordinate hyperplanes in the affine $d$ space) and given a finite dimensional complex representation of the fundamental group $\pi_{1}(U)$, Deligne constructed a system of differential equations with regular singular points (in an appropriately generalized sense) whose solutions via continuation along paths present the given monodromy. The system is essentially unique. If $X$ is complete (compact), then the equations are algebraic.

Deligne came to the problem from his work on monodromy, in particular that on Picard-Lefschetz theory, which Grothendieck had encouraged between 1967 and 1969 as the next step toward the proof of the remaining Weil conjecture, the Weil-E. Artin-Riemann hypothesis. He drew further inspiration from the work of Michael Atiyah and William Hodge and the work of Grothendieck on the case of the trivial representation and of a number of people on the Gauss-Manin connection (system). The importance of Deligne's contribution to the subject of the Riemann-Hilbert problem cannot be overestimated; it inspired and supported all the subsequent advances.

Around 1977, a definitive generalization of the Riemann-Hilbert problem was formulated. In 1979, that generalization was solved by Zoghman Mebkhout [79] and, in 1980, by Masaki Kashiwara [54] somewhat differently. Both of those treatments are analytic. In the fall of 1980, Alexandre Beilinson and Joseph Bernstein developed a purely algebraic treatment, which is sufficient for the proof of the Kazhdan-Lusztig conjecture. It is largely analogous to the analytic treatment, but is often technically simpler. See [6, p. 328, bot.].

To pass to the generalization, first view the monodromy representation in an equivalent form, as a locally constant sheaf of finite dimensional complex vector spaces on $U$. Then equip $X$ with a Whitney stratification, and let the sheaf be an arbitrary constructible sheaf, or better a bounded complex of sheaves whose cohomology sheaves are constructible.

The definitive generalization does not directly involve any system of differential equations $A F=0$ where $A$ is an $m$ by $n$ matrix of linear partial differential operators and $F$ is a vector of meromorphic functions $y(z)$ on $X$. Rather, it deals with the associated (left) $\mathcal{D}$-module $\mathcal{M}$ defined by a presentation

$$
\mathcal{D}^{m} \stackrel{A^{T}}{\longrightarrow} \mathcal{D}^{n} \longrightarrow \mathcal{M} \longrightarrow 0
$$

where $A^{T}$ denotes the operation of right multiplication with the matrix $A$. That change is reasonable because applying the functor $\operatorname{Hom}_{\mathcal{D}}\left(\cdot, \mathcal{O}_{X}\right)$ to the presentation yields this exact sequence:

$$
0 \longrightarrow \operatorname{Hom}_{\mathcal{D}}\left(\mathcal{M}, \mathcal{O}_{X}\right) \longrightarrow \mathcal{O}_{X}^{n} \stackrel{A}{\longrightarrow} \mathcal{O}_{X}^{m} .
$$


So the sheaf of local solutions is $\operatorname{Hom}_{\mathcal{D}}\left(\mathcal{M}, \mathcal{O}_{X}\right)$ and thus depends only on $\mathcal{M}$. There is a further reasonable change: the $\mathcal{D}$-module $\mathcal{M}$ is required to have such a presentation only locally. Such an $\mathcal{M}$ is termed coherent. (The term is reasonable because $\mathcal{D}$ is left Noetherian.)

The characteristic variety, or singular support, of a coherent $\mathcal{D}$-module $\mathcal{M}$ is a (reduced) closed subvariety of the cotangent bundle $T^{*} X$. It is denoted by $\mathrm{Ch}(\mathcal{M})$, or $S . S(\mathcal{M})$, and is defined locally as follows: filter $\mathcal{M}$ by the image of the filtration on $\mathcal{D}^{n}$ by operator order; then the associated graded module $\operatorname{Gr}(\mathcal{N})$ is finitely generated over the associated graded ring $\mathcal{G} r(\mathcal{D})$, and $\mathcal{G} r(\mathcal{D})$ is equal to the direct image on $X$ of the structure sheaf of $T^{*} X$; set

$$
\operatorname{Ch}(\mathcal{M}):=\operatorname{Supp}(\mathcal{G r}(\mathcal{M})) \text {. }
$$

Then each component of $\operatorname{Ch}(\mathcal{M})$ has dimension at least $d$ where $d:=\operatorname{dim}(X)$. In fact, each component comes with a natural multiplicity of appearance, the length of $\operatorname{Gr}(\mathcal{N})$ at a general point of the component. The corresponding characteristic cycle will also be denoted by $\mathrm{Ch}(\mathcal{M})$.

A $\mathcal{D}$-module $\mathcal{M}$ is called holonomic if it is coherent and if its characteristic variety $\operatorname{Ch}(\mathcal{M})$ is of (pure) dimension $d$. Then the solution sheaf and its satellites, the sheaves $\operatorname{Ext}_{\mathcal{D}}^{i}\left(\mathcal{N}, \mathcal{O}_{X}\right)$, are constructible with respect to some Whitney stratification.

A holonomic module $\mathcal{M}$ is said to have regular singular points or, simply, to be regular, if every formal generalized local solution is convergent, that is, if, for every $x \in X$ and every $i$,

$$
\operatorname{Ext}_{\mathcal{D}}^{i}\left(\mathcal{M}, \mathcal{O}_{X}\right)_{x}=\operatorname{Ext}_{\mathcal{D}_{x}}^{i}\left(\mathcal{M} \widehat{\mathcal{O}}_{x}\right),
$$

where $\widehat{\mathcal{O}}_{x}$ is the ring of formal power series at $x$. Other definitions are also used. In any case, $\mathcal{M}$ is regular if and only its pullback to any (smooth) curve mapping into $X$ is regular. For a curve, the modern concept is equivalent to Fuchs's.

The dual of a holonomic $\mathcal{D}$-module $\mathcal{M}$ is, by definition, the $\mathcal{D}$-module

$$
{ }^{*} \mathcal{M}:=\mathcal{H}_{\operatorname{oom}_{\mathcal{O}_{X}}}\left(\Omega_{X}^{d}, \mathbf{E x t}_{\mathcal{D}}^{d}(\mathcal{M}, \mathcal{D})\right)=\operatorname{Ext}_{\mathcal{D}}^{d}\left(\mathcal{M}, \mathcal{D}^{\Omega}\right)
$$

where $\Omega_{X}^{d}$ is the sheaf of holomorphic $d$-forms, $d:=\operatorname{dim}(X)$, and

$$
\mathcal{D}^{\Omega}:=\mathcal{D} \otimes\left(\Omega_{X}^{d}\right)^{-1}=\mathcal{H}_{\operatorname{Com}_{\mathcal{O}_{X}}}\left(\Omega_{X}^{d}, \mathcal{D}\right) .
$$

Then ${ }^{*} \mathcal{M}$ is holonomic, and ${ }^{*} \mathcal{M}=\mathcal{M}$. If $\mathcal{M}$ is regular, so is ${ }^{*} \mathcal{M}$. Moreover, $\mathcal{O}_{X}$ is holonomic (its characteristic variety is the zero-section), and ${ }^{*} \mathcal{O}_{X}=\mathcal{O}_{X}$.

The definitive generalization of the Riemann-Hilbert problem involves bounded complexes $\mathcal{M}$ of $\mathcal{D}$-modules whose cohomology sheaves are regular holonomic $\mathcal{D}$-modules. The duality above, $\mathcal{M} \mapsto{ }^{*} \mathcal{M}$, extends to these complexes, viewed in the derived category. To such a complex $\mathcal{N}$, are associated the following two 
complexes in the derived category of bounded complexes of sheaves of $\mathbb{C}$-vector spaces:

$$
\begin{aligned}
\operatorname{Sol}(\mathcal{M}) & :=\operatorname{RHom}_{\mathcal{D}}\left(\mathcal{M}, \mathcal{O}_{X}\right) \\
\operatorname{deR}(\mathcal{M}) & :=\operatorname{RHom}_{\mathcal{D}}\left(\mathcal{O}_{X}, \mathcal{M}\right) .
\end{aligned}
$$

The first complex, $\operatorname{Sol}(\mathcal{M})$, is the complex of generalized solutions; its cohomology sheaves are the solution sheaf and its satellites, $\operatorname{Ext}_{\mathcal{D}}^{i}\left(\mathcal{M}, \mathcal{O}_{X}\right)$. The second complex, $\operatorname{deR}(\mathcal{M})$, is isomorphic (in the derived category) to the complex

$$
0 \rightarrow \mathcal{M} \rightarrow \Omega_{X}^{1} \otimes_{\mathcal{O}_{X}} \mathcal{M} \rightarrow \cdots \rightarrow \Omega_{X}^{d} \otimes_{\mathcal{O}_{X}} \mathcal{M} \rightarrow 0,
$$

and so it is called the deRham complex of $\mathcal{M}$. The two complexes are related through duality and the following two key canonical isomorphisms:

$$
\operatorname{Sol}\left({ }^{*} \mathcal{M}\right)=\operatorname{deR}(\mathcal{M})=\operatorname{Sol}(\mathcal{M})^{\sim}
$$

where the " $"$ ' indicates the Verdier-Borel-Moore dual.

The definitive generalization of the Riemann-Hilbert problem may be stated now. The problem is to prove the following theorem, which describes the nature of the correspondence between a system of differential equations and its solutions.

Theorem (Riemann-Hilbert correspondence) [68], [6]. Given a bounded complex of sheaves of complex vector spaces $\mathbf{S}$ whose cohomology sheaves are constructible with respect to a fixed Whitney stratification of $X$, there exists a bounded complex $\mathcal{M}$ of $\mathcal{D}$-modules, unique up to isomorphism in the derived category, such that (1) its cohomology sheaves $\mathcal{H}^{i}(\mathcal{N})$ are regular holonomic $\mathcal{D}$-modules whose characteristic varieties are contained in the union of the conormal bundles of the strata, and (2) the solution complex $\mathbf{S o l}(\mathcal{M})$ is isomorphic to $\mathbf{S}$ in the derived category. Moreover, the functor

$$
\mathcal{M} \mapsto \operatorname{Sol}(\mathcal{M})
$$

is an equivalence between the derived categories, which commutes with direct image, inverse image, exterior tensor product, and duality.

The Kazhdan-Lusztig conjecture was proved during the summer and fall of 1980 independently and in essentially the same way by Beilinson and Bernstein in Moscow and by Jean-Luc Brylinski and Kashiwara in Paris. Earlier, in 1971, Bernstein, I. Gelfand, and S. Gelfand had considered a complex semisimple Lie algebra $\mathfrak{g}$, and constructed a resolution by Verma modules $M_{\lambda}$ of the irreducible module $L_{\mu}$ with a positive highest weight $\mu$. In April 1976, George Kempf had given a geometric treatment of the resolution, and Kempf's work provided some initial inspiration for both proofs. Beilinson and Bernstein discussed intersection homology theory with MacPherson during his stay in Moscow for the first six months of 1980. By the middle of September, they had proved the conjecture [2]. 
Brylinski had become seriously interested in the conjecture in the fall of 1979 and, over the next nine months, he filled in his background. In early June 1980, while reading someone else's notes from a two-day conference that May on $\mathcal{D}$ module theory, he suddenly realized that that theory was the key to proving the conjecture. Shortly afterwards, he attended a lecture of Lê's and told him his ideas. Lê gave him his personal notes from some lectures of Mebkhout and encouraged Brylinski to phone him. Instead of phoning, Brylinski got a hold of Mebkhout's thesis and some articles by Kashiwara and Takahiro Kawai. On July 21, 1980, he wrote up a ten-page program of proof and sent it to a half dozen people; the main problem was to establish the regularity asserted in the following lemma. Soon afterwards, Kashiwara phoned him, saying he wanted to talk about it. They collaborated several times in July and August and, by the middle of September, they had written up a first draft of their proof. The proof was announced in [14] and presented in [15].

The main lemmas used in the proof of the Kazhdan-Lusztig conjecture are these.

Lemma $[94,3.7,3.8]$. Let $\mathcal{O}_{\text {triv }}$ denote the (Bernstein-Gelfand-Gelfand) category of representation modules $M$ such that (1) $M$ is finitely generated over the universal enveloping algebra $U$ of the complex semisimple Lie algebra $\mathfrak{g}$, (2) any $m \in M$ and its translates under the action of the enveloping algebra of a Borel subalgebra form a finite dimensional vector space, and (3) the center of $U$ acts trivially on $M$. Then the functor $M \mapsto \mathcal{D}_{X} \otimes M$, where $X$ is the flag manifold, defines an equivalence of the category $\mathcal{O}_{\text {triv }}$ with the category of regular holonomic $\mathcal{D}_{X}$ modules $\mathcal{M}$ whose characteristic variety is contained in the union of the conormal bundles of the Bruhat cells $B_{w}$; the inverse functor is $\mathcal{M} \mapsto \Gamma(X, \mathcal{M})$.

Lemma $[94,3.15,3.16]$. Let $\mathbb{C}_{w}$ denote the extension by 0 of the constant sheaf on $\mathbb{C}$ on the Bruhat cell $B_{w}$. Consider the Verma module $M_{w}:=M_{-\rho y-\rho}$ and its simple quotient $L_{w}:=L_{-\rho y-\rho}$. Set $d:=\operatorname{dim}(X)$. Then

$$
\begin{aligned}
\operatorname{deR}\left(\mathcal{D}_{X} \otimes M_{w}\right) & =\mathbb{C}_{w}[l(w)-d] \\
\operatorname{deR}\left(\mathcal{D}_{X} \otimes L_{w}\right) & =\mathbf{I C} \cdot\left(X_{w}\right)[l(w)-d]
\end{aligned}
$$

where the right sides are the shifts down by $l(w)-d$ of the complex consisting of the sheaf $\mathbb{C}_{w}$ concentrated in degree 0 and of the intersection cohomology complex of the Schubert variety $X_{w}$, the closure of $B_{w}$.

The second formula of the last lemma is proved by checking the axioms that characterize IC $\left(X_{w}\right)$.

The first formula implies by additivity that for any $M \in \mathcal{O}_{\text {triv }}$ the cohomology sheaves of the deRham complex $\operatorname{deR}\left(\mathcal{D}_{X} \otimes M\right)$ are locally constant with finite dimensional stalks on any cell $B_{w}$. Hence it is meaningful to consider the "index,"

$$
\chi_{w}(M):=\sum_{i}(-1)^{i} \operatorname{dim}_{\mathbb{C}} \mathbf{H}^{i}\left(\operatorname{deR}\left(\mathcal{D}_{X} \otimes M\right)\right)_{w},
$$


where the subscript ' $w$ ' indicates the stalk at the base point of $B_{w}$. For example,

$$
\chi_{w}\left(M_{y}\right)=(-1)^{l(w)-d} \delta_{w y}
$$

by the first formula, where $\delta_{w y}$ is the Kronecker function. The first formula and additivity now yield the formula,

$$
M=\sum_{y}(-1)^{d-l(y)} \chi_{y}(M) M_{y},
$$

in the Grothendieck group. Finally, the second formula yields the first formula in the Kazhdan-Lusztig conjecture and, as Kazhdan and Lusztig showed, their second formula is formally equivalent to the first.

\section{PeRverse SheAves}

Beilinson and Bernstein had succeeded in proving the Kazhdan-Lusztig conjecture when Deligne arrived in Moscow in mid-September 1980. The three of them discussed the proof and its implications. There is, they realized, a natural abelian category inside the nonabelian 'constructible derived category'the derived category of bounded complexes $\mathbf{S}$ of sheaves of complex vector spaces whose cohomology sheaves $\mathbf{H}^{i}(\mathbf{S})$ are constructible. It is just the essential image of the category of regular holonomic $\mathcal{D}$-modules $\mathcal{M}$ embedded by the RiemannHilbert correspondence, $\mathbf{S}=\operatorname{deR}(\mathcal{M})$. It exists on any smooth complex algebraic variety $X$. Now, how can this unexpected abelian subcategory be characterized intrinsically?

Ironically, around Easter the year before, 1979, Deligne and Mebkhout had chatted in Paris about the Riemann-Hilbert correspondence. Mebkhout had just established it in his thesis [79], and Lê, then in Stockholm, wrote to Mebkhout and urged him to go and talk to Deligne about it. However, Deligne said politely that, while the subject was very interesting, nevertheless it appeared to be far removed from his work [29] in progress on monodromy, pure complexes, and the hard Lefschetz theorem. That was also the time of Deligne's correspondence with Kazhdan and Lusztig about their conjecture.

In the middle of October 1980, Deligne returned to Paris. MacPherson was there and became excited on hearing about that abelian subcategory; he kept asking Deligne if its existence was not a topological fact. The question had been discussed, according to Beilinson, by Bernstein, Deligne and himself while Deligne was still in Moscow. The time was right, and Deligne soon proved the following theorem, based on those discussions, which characterizes that image category topologically.

Theorem [10, §1]. Given a bounded complex $\mathbf{S}$ with constructible cohomology sheaves $\mathbf{H}^{i}(\mathbf{S})$ on an arbitrary smooth complex algebraic variety $X$, there exists a regular holonomic $\mathcal{D}$-module $\mathcal{M}$ such that $\mathbf{S} \cong \operatorname{deR}(\mathcal{M})$ in the derived category if and only if both of the following dual conditions are satisfied: 
(i) $\mathbf{H}^{i}(\mathbf{S})=0$ for $i<0$ and $\operatorname{codim}\left(\operatorname{Supp}\left(\mathbf{H}^{i}(\mathbf{S})\right)\right) \geq i$ for $i \geq 0$,

(i`) $\mathbf{H}^{i}\left(\mathbf{S}^{\ulcorner}\right)=0$ for $i<0$ and $\operatorname{codim}\left(\operatorname{Supp}\left(\mathbf{H}^{i}\left(\mathbf{S}^{\complement}\right)\right)\right) \geq i$ for $i \geq 0$, where $\mathbf{S}^{\sim}$ is the Verdier-Borel-Moore dual of $\mathbf{S}$.

Conditions (i) and (i`) were not far-fetched; a condition like (i) had appeared in [90, XIV 3], and Deligne [29, 6.2.13] had generalized the hard Lefschetz theorem to a pure complex $\mathbf{S}$ satisfying (i) and (i־); see $\S 7$. The technical aspect of the proof was not that difficult. Indeed, if $\mathbf{S}=\operatorname{deR}(\mathcal{M})$, then $\mathbf{S}=\operatorname{Sol}\left(\mathcal{M}^{*}\right)$ and $\mathbf{S}=\mathbf{S o l}(\mathcal{N})$ by Mebkhout's local duality theorems [80, Thm.1.1, Ch. III]; hence, (i) and ( $\mathrm{i}^{`}$ ) hold by Kashiwara's Thm. 4.1 of [53]. Conversely, if (i) and (i) hold, then it can be proved, via a 'dévissage', that a complex $\mathcal{M}$ such that $\mathbf{S} \cong \operatorname{deR}(\mathcal{M})$ has cohomology only in degree 0 . Independently, according to [10, footnote on p. 2], Kashiwara too discovered that theorem.

Deligne had the right perspective, so he proved more of what he, Beilinson, and Bernstein had conjectured together in Moscow. The conditions (i) and (i־) of the theorem above define a full abelian subcategory also if $X$ is an algebraic variety in arbitrary characteristic $p$ with the étale topology. The conditions can be modified using an arbitrary perversity so that they still yield a full abelian subcategory. Moreover, unlike arbitrary complexes in the derived category, those $\mathbf{S}$ that satisfy the modified conditions can be patched together from local data like sheaves. The original conditions (i) and (i־) are recovered with the middle perversity. The case of the middle perversity is once again the most useful by far because of the additional theorems that hold in it, such as the next two theorems. It is the only case that will be considered from now on.

Because of all those marvelous properties, everyone calls these special complexes $\mathbf{S}$ (or sometimes, their shifts by $d:=\operatorname{dim}(X)$ ) perverse sheaves. Of course, they are complexes in a derived category and are not sheaves at all. Moreover, they are well behaved and are not perverse at all. Nevertheless, despite some early attempts to change the name 'perverse sheaf', it has stuck.

Theorem [3, 4.3.1(i)]. The abelian category of perverse sheaves is Noetherian and Artinian: every object has finite length.

Theorem [3, 4.3.1(ii)]. Let $V$ be a smooth, irreducible locally closed subvariety of codimension $c$ of $X$, and $\mathbf{L}$ a locally constant sheaf of vector spaces on $V$.

(1) There is a unique perverse sheaf $\mathbf{S}$ whose restriction to $V$ is equal to $\mathbf{L}[-c]$, which is the complex that consists of $\mathbf{L}$ concentrated in degree $c$.

(2) If $\mathbf{L}$ is the constant sheaf with 1-dimensional stalks, then $\mathbf{S}$ is equal to the shifted intersection homology complex $\mathbf{I C} \cdot(\bar{V})[-c]$, where $\bar{V}$ is the closure of $V$. In general, $\mathbf{S}$ can be constructed from $\mathbf{L}$ by the same process of repeated pushforth and truncation.

(3) If $\mathbf{L}$ is an irreducible locally constant sheaf, then $\mathbf{S}$ is a simple perverse sheaf. Conversely, every simple perverse sheaf has this form. 
The perverse sheaf $\mathbf{S}$ of the last theorem is denoted $\mathbf{I C} \cdot(\bar{V}, \mathbf{L})[-c]$ and is called the DGM extension, or Deligne-Goresky-MacPherson extension, of $\mathbf{L}$. It is also called the "twisted intersection cohomology complex with coefficients in L." Thus the family of intersection cohomology complexes was enlarged through twisting and then forever abased, becoming merely the family of simple objects in the magnificent new abelian category of perverse sheaves.

The moment that Deligne told MacPherson the definition of a perverse sheaf, MacPherson realized that some work that he and Goresky had done about three years earlier implied that a perverse sheaf 'specializes' to a perverse sheaf. Indeed, earlier they had thought hard about the way that the intersection cohomology complex specializes. They were rather upset to find that the middle perversity complex did not specialize to the middle perversity complex but to the complex associated to the next larger perversity, which they called the logarithmic perversity. Even worse, the logarithmic perversity complex also specialized to the logarithmic complex. The explanation turned out now to be simple: both complexes are perverse sheaves, and the logarithmic complex is in some sense a "terminal" object in the category of perverse sheaves. Goresky and MacPherson's main result in that connection is this.

Theorem (Specialization) $[43, \S 6]$. In a 1-parameter family, a perverse sheaf specializes to a perverse sheaf. More precisely, if $S$ is an algebraic curve, $s \in S$ a simple point, $f: X \rightarrow S$ a map, $X_{s}:=f^{-1}(s)$ the fiber, and $\mathbf{S}$ a perverse sheaf on $X-X_{s}$, then the shifted complex of 'nearby cycles' $\mathbf{R} \Psi_{f} \mathbf{S}[-1]$, which is supported on $X_{s}$, is a perverse sheaf on $X$. Moreover, the functor $\mathbf{R} \Psi_{f}$ commutes with Verdier-Borel-Moore duality.

Goresky and MacPherson used special techniques from stratification theory to construct a neighborhood $U$ of $X_{s}$ and a continuous retraction $\Psi: U \rightarrow X_{s}$ that is locally trivial over each stratum of $X_{s}$. Then they defined $\mathbf{R} \Psi_{f} \mathbf{S}$ by the equation

$$
\mathbf{R} \Psi_{f} \mathbf{S}:=\mathbf{R} \Psi_{*} \iota_{t}^{*} \mathbf{S}
$$

where $t \in S$ is a nearby general point and $\iota_{t}: X_{t} \rightarrow X$ is the inclusion. They proved that $\mathbf{R} \Psi_{f} \mathbf{S}$ is independent of the choice of the stratification and the retraction. Thus $\mathbf{R} \Psi_{f} \mathbf{S}$ is clearly constructible. They established the support conditions (i) and ( $\mathrm{i}^{\sim}$ ) using their stratified Morse theory.

During the next year, MacPherson told most everyone he met about the specialization theorem. Of course, it has a natural statement, and some people may have thought of it themselves. At any rate, it became well known. It was reproved by Bernard Malgrange, by Kashiwara, and by Bernstein using $\mathcal{D}$-module theory. It was proved in arbitrary characteristic, using Deligne's 1968 algebraic definition of $\mathbf{R} \Psi_{f} \mathbf{S}$, by Gabber and by Beilinson and Bernstein. Verdier [97] considered the case of specialization to a divisor that is not necessarily principal. At the time, the sheaf $\mathbf{R} \Psi_{f} \mathbf{S}$ was often (improperly) called the sheaf of 'vanishing cycles'. 
The "true" perverse sheaf $\mathbf{R} \Phi_{f} \mathbf{S}$ of vanishing cycles is defined when the perverse sheaf $\mathbf{S}$ is given on all of $X$. It is defined as the mapping cone over the natural comparison map,

$$
\iota_{s}^{*} \mathbf{S}[-1] \rightarrow \mathbf{R} \Psi_{f} \mathbf{S}[-1],
$$

where $\iota_{s}: X_{s} \rightarrow X$ is the inclusion. Thus it is a measure of the difference between the nearby cycles and the cycles on the special fiber $X_{s}$. Deligne conjectured the following remarkable theorem, which enumerates the vanishing cycles.

Theorem [67, (1.5), (4.1)]. Choose a local parameter at $s \in S$, and consider the corresponding section $d f$ of the cotangent bundle $T^{*} X$. Let $\mathcal{M}$ be a regular holonomic $\mathcal{D}$-module such that $\mathbf{S} \cong \operatorname{deR}(\mathcal{M})$, and suppose that the characteristic cycle $\mathrm{Ch}(\mathcal{M})$ and the section $d f$ have an isolated intersection at a point $\xi$ of $T^{*} X$ outside the 0 -section and lying over a point $x \in X$. Then the support (of every cohomology sheaf) of $\mathbf{R} \Phi_{f} \mathbf{S}$ is isolated at $x$, and

$$
\operatorname{dim}\left(\mathbf{H}^{i}\left(\mathbf{R} \Phi_{f} \mathbf{S}\right)_{x}\right)= \begin{cases}\operatorname{mult}_{\xi}(\operatorname{Ch}(\mathcal{M}) \cdot[d f]), & \text { if } i=n ; \\ 0, & \text { otherwise. }\end{cases}
$$

The assertion about the support of the complex $\mathbf{R} \Phi_{f} \mathbf{S}$ results directly from the description of the complex given in January 1983 by Lê and Mebkhout [69, Prop. 2.1]. The formula for the dimension was first proved by Lê at Luminy in July 1983, but that proof required a condition on the restriction of $f$ to the variety $\mathrm{Ch}(\mathcal{M})$. In January 1988, Lê [67] eliminated this requirement via a more profound topological analysis inspired by some work that he did with Mitsuyoshi Kato in 1975. Meanwhile, Claude Sabbah (1985) and V. Ginzburg (1986) gave proofs based on an interesting calculus of 'Lagrangian cycles'. A related proof was sketched earlier (1984) by Alberto Dubson but, according to Lê [67, (4.1.3)], he stated a crucial and delicate step without sufficient justification.

In March of 1981, MacPherson went to Moscow and brought along a copy of Deligne's manuscript on perverse sheaves. It turned out that the previous fall Beilinson and Bernstein had worked out an elementary theory of algebraic $\mathcal{D}$-modules and that independently they too had begun to develop the theory of perverse sheaves. When MacPherson mentioned the specialization theorem, Beilinson and Bernstein immediately sat down and came up with their own proof. Then their work became stranded, when all of a sudden Bernstein was granted permission to emigrate. Their theory of algebraic $\mathcal{D}$-modules was later written up and published by Borel et al. [6].

The two developments of the theory of perverse sheaves were combined by Beilinson, Bernstein, and Deligne, and published in their monograph [3], which is the definitive work on perverse sheaves in arbitrary characteristic. It includes the only detailed account of the comparison of the theories in the classical topology and in the étale topology over $\mathbb{C}$ and the only detailed account of the reduction 
to the algebraic closure of a finite field. In addition to discussing the theorems already mentioned and some others, which are considered in the next section, the monograph [3] touches on some more issues of monodromy and vanishing cycles. Parts of the monograph are rather sophisticated and based on some of Gabber's ideas. Gabber should properly have been a fourth co-author, but he declined at the last moment.

MacPherson and Kari Vilonen, another of MacPherson's students, after conversations with Beilinson and Deligne, gave in [77] and [78] another construction of the category of perverse sheaves on a stratified topological space with only even (real) dimensional strata $X_{i}-X_{i-1}$. It proceeds recursively, passing from $X-X_{i}$ to $X-X_{i+1}$. That construction makes the structure of the category more concrete. Previously, a number of other authors had made similar constructions in various special cases - dimension 2, strata with normal crossings, etc. More recently, Beilinson [1] gave a short alternative treatment in the general case. Renato Mirollo and Vilonen [82] used the construction of MacPherson and Vilonen to extend the results of Bernstein, I. Gelfand, and S. Gelfand about the Cartan matrix of the category $\mathcal{O}_{\text {triv }}$ (see the end of $\S 5$ ) to the category of perverse sheaves on a wide class of complex analytic spaces.

\section{Purity AND DECOMPosition}

About July 1980, Gabber solved the problem of purity that Deligne posed in his letter to Kazhdan and Lusztig. In fact, he proved more. The precise statement requires some terminology, which was introduced in $[29,1.2 .1,1.2 .2$, 6.2.2, and 6.2.4] and reviewed in [3, 5.1.5 and 5.1.8]. An $l$-adic sheaf $\mathbf{F}$ on an algebraic variety $X$ defined over the field with $q$-elements is called punctually pure of weight $w$ if, for every $n$ and for every fixed-point $x$ of the Frobenius endomorphism $\phi_{q^{n}}: X \rightarrow X$, the eigenvalues of the automorphism $\phi_{q^{n}}^{*}$ of $\mathbf{F}_{x}$ are algebraic numbers whose complex conjugates all have absolute value exactly $\left(q^{n}\right)^{w / 2}$. The sheaf $\mathbf{F}$ is called mixed if it admits a finite filtration whose successive quotients are punctually pure; the weights of the nonzero quotients are called the punctual weights of $\mathbf{F}$. A complex of $l$-adic sheaves $\mathbf{S}$ is called mixed of weight at most $w$ if for each $i$ the cohomology sheaf $\mathbf{H}^{i}(\mathbf{S})$ is mixed with punctual weights at most $w$. Finally, $\mathbf{S}$ is called pure of weight $w$ if $\mathbf{S}$ is mixed of weight at most $w$ and if its Verdier-Borel-Moore dual $\mathbf{S}^{\sim}$ is mixed of weight at most $-w$.

Gabber's theorem is this.

Theorem (Purity) [29, p. 251], [11, 3.2], [3, 5.3]. If $X$ is an algebraic variety over the algebraic closure of a finite field, then the intersection homology complex IC $(X)$ is pure of weight 0 ; in fact, any DGM extension $\mathbf{I C} \cdot(\bar{V}, \mathbf{L})[-c]$ is pure of weight $-c$. 
The theorem shows in particular that there are unexpectedly many pure complexes to which to apply Deligne's theory [29].

In the fall of 1980, Gabber and Deligne collaborated to prove some key lemmas about the structure of pure complexes and mixed perverse sheaves and to derive some important consequences. Independently, Beilinson and Bernstein obtained the same results. All the details were presented in the combined treatise [3]. The theory is based on Deligne's work on the Weil conjectures [29] and [28], which in turn is supported by over 3000 pages on étale cohomology theory [90], [91], on $l$-adic cohomology theory and $L$-functions [92], and on monodromy [93]. Thus these results are some of the deepest theorems in algebraic geometry, if not all of mathematics.

The Weil-E. Artin-Riemann hypothesis and the hard Lefschetz theorem, which were discussed near the end of $\S 4$, are two major consequences of the purity theorem. They hold for a projective variety defined over an algebraically closed field; for the Riemann hypothesis, it must be the algebraic closure of a finite field, but for the Lefschetz theorem, it may be arbitrary, it may even be the field of complex numbers $\mathbb{C}$ ! Over $\mathbb{C}$, an analytic proof of the Lefschetz theorem, based on a theory of "polarizable Hodge modules" analogous to the theory of pure perverse sheaves, was given by Morihiko Saito [84] and [85].

One lovely application in intersection theory in algebraic geometry of the hard Lefschetz theorem was made by Fulton and Robert Lazarsfeld; they used it to give a significantly shorter proof, which moreover is valid in arbitrary characteristic, of the following theorem of Spencer Bloch and David Gieseker.

Theorem [31]. Let $X$ be a projective variety of dimension $d$, and $E$ an ample vector bundle of rank $e$ on $X$. If $e \geq n$, then

$$
\int_{X} c_{n}(E)>0 .
$$

Doubtless, the single most important consequence of the purity theorem is the following theorem.

Theorem (Decomposition) [11, 3.2.3], [3, 6.2.5]. If $f: X \rightarrow Y$ is a proper map of varieties in arbitrary characteristic, then $\mathbf{R} f_{*} \mathbf{I C} \cdot(X)$ is a direct sum of shifts of DGM extensions $\mathbf{I C} \cdot\left(\bar{V}_{i}, \mathbf{L}_{\mathbf{i}}\right)\left[-e_{i}\right]$, where $e_{i}$ is not necessarily the codimension of $V_{i}$.

Indeed, IC $(X)$ is of 'geometric origin', so it suffices to prove the theorem when $X, Y$ and $f$ are defined over the algebraic closure of a finite field. Then IC $(X)$ is pure by the purity theorem. It therefore follows from Deligne's main theorem [29, 6.2.3] that $\mathbf{R} f_{*} \mathbf{I C} \cdot(X)$ is pure. Finally, because an eigenvalue of the Frobenius automorphism whose weight is nonzero cannot be equal to 1 , it can be proved that certain Ext $^{1}$ 's must vanish and so the corresponding extensions must split. 
The decomposition theorem was conjectured in the spring of 1980 by Sergei Gelfand and MacPherson [35, 2.10], then proved that fall by Gabber and Deligne and independently by Beilinson and Bernstein. Over $\mathbb{C}$, an analytic proof was given several years later by Morihiko Saito in [84] and [85]. In fact, more general versions of the theorem are proved in each case: IC $(X)$ is replaced by the DGM extension of a locally constant sheaf of a certain fairly general type.

Some implications of the decomposition theorem are discussed by Goresky and MacPherson in [41]. In particular, they say in $\S 2$ that if the $V_{i}$ and $\mathbf{L}_{i}$ are taken to be irreducible (as they may be), then the summands $\mathbf{I C} \cdot\left(\bar{V}_{i}, \mathbf{L}_{\mathbf{i}}\right)\left[-e_{i}\right]$ and their multiplicities of appearance are uniquely determined. However, the full derived category is not abelian, and the decomposition is in no sense canonical by itself. On the other hand, Deligne has observed, see [76, §12] and [64, 4.2, (iii)-(v)], that the decomposition can be made canonical with respect to a relatively ample sheaf if $f$ is projective.

Sergei Gelfand and MacPherson [35, 2.12] showed that the decomposition theorem yields the main theorem of Kazhdan and Lusztig, which relates their polynomials to the intersection homology groups of the Schubert varieties (see also $[94,2])$. This derivation involves a lovely interpretation of the Hecke algebra as an algebra of correspondences. Moreover, given the decomposition theorem over $\mathbb{C}$, the proof involves no reduction to positive characteristic. According to [94, 2.13], similar work was done independently by Beilinson and Bernstein, by Brylinski, and by Lusztig and Vogan [75, 5]. In fact, the latter two authors considered a more general situation, in which the Schubert varieties are replaced by the orbits of the centralizer of an involution. However, all these latter authors used the purity theorem directly rather than applying the decomposition theorem, probably because they were unaware of it at the time. In addition, in [2], Beilinson and Bernstein also treated the case of Verma modules with regular rational highest weight, showing that again there is a topological interpretation for the multiplicities in the Jordan-Hölder series in terms of intersection homology groups. Lusztig [73] carried that work further, giving some explicit formulas and applying the results to the classification of the irreducible representations of the finite Chevalley groups; Lusztig's work rests on both the purity theorem and the decomposition theorem.

The decomposition theorem has the following rather useful corollary, which Kazhdan had conjectured in 1979.

Corollary [11, 3.2.5]. If $f: X \rightarrow Y$ is a resolution of singularities, then $I_{i}(Y)$ is a direct summand of $H_{i}(X)$. In fact, then $\mathbf{I C} \cdot(Y)$ is a direct summand of $\mathbf{R} f_{*} \mathbb{Q}_{l, X}$.

Goresky and MacPherson in $[41, \S \mathrm{A}]$ gave two examples showing that the direct sum decomposition need not be canonical. Nevertheless, it follows, for 
instance, that if $H_{i}(X)=0$, then $I H_{i}(Y)=0$. Thus the odd dimensional intersection homology groups of $Y$ vanish if $Y$ is a Schubert variety or if $Y$ is an orbit closure in the product of a flag manifold with itself; for a proof, see Roy Joshua's paper [51, (3)].

If $Y$ is the toric variety associated to a simplicial $d$-polytope, then it follows similarly that $I H_{i}(Y)=0$ for odd $i$. Richard Stanley [95] used that fact to prove this: the components $h_{i}$ of the $h$-vector of an arbitrary rational $d$-polytope are nonnegative; in fact, $h_{i}=\operatorname{dim}\left(I H_{2(d-i)}(Y)\right)$ for a suitable toric variety $Y$. Stanley went on to observe that, because of the hard Lefschetz theorem, the vector is unimodal and the generalized Dehn-Sommerville equations are satisfied:

$$
1=h_{0} \leq h_{1} \leq \ldots \leq h_{[d / 2]} \quad \text { and } \quad h_{i}=h_{d-i} .
$$

The equations are obviously also a consequence of Poincaré duality.

Frances Kirwan [60] used the last corollary and the hard Lefschetz theorem to establish a procedure for computing the dimensions of the rational intersection homology groups of the quotient assigned by David Mumford's geometric invariant theory (1965) to a linear action of a complex reductive group on a smooth complex projective variety $X$. Just before, Kirwan had published a systematic procedure for blowing up $X$ along a sequence of smooth equivariant centers to obtain a variety $\widetilde{X}$ such that every semi-stable point of $\widetilde{X}$ is stable. Then the quotient of $\widetilde{X}$ is a partial desingularization of the quotient of $X$ in which the more serious singularities have been resolved; in fact, the quotient of $\widetilde{X}$ is topologically just the ordinary quotient of the open set of semi-stable points $\widetilde{X}^{s s}$, and it is everywhere locally isomorphic to the quotient of a smooth variety by a finite group. Hence the intersection homology groups of the latter quotient are equal to its ordinary homology groups. Moreover, they are also equal to the equivariant homology groups of $\widetilde{X}^{s s}$, whose dimensions were computed in another of Kirwan's papers. The heart of [60] is a description of the change in the intersection homology groups under the passage to the next successive blow-up. In the sequel [61], Kirwan generalized the work to the case in which $X$ is singular.

Kirwan [62] used the last corollary and Heisuke Hironaka's (1976) equivariant resolution of singularities to treat the rational intersection homology groups of a singular complex projective variety $Y$ with a torus action. The groups are determined by the action of the torus on an arbitrarily small neighborhood of the set of fixed points, and they are given by a generalization of a well-known direct sum formula. Thus Kirwan's results generalize the results of Andre Bialynicki-Birula $(1973,1974)$ in the case that $Y$ is smooth and the results of James Carrell and Goresky (1983) in the case that $Y$ is singular but its Bialynicki-Birula decomposition is suitably "good." Kirwan also discussed a supplementary treatment using an equivariant intersection homology theory. In that discussion, Kirwan referred to the treatments of equivariant intersection homology theory made by 
Brylinski [13] and Joshua [51]. However, all three treatments of the equivariant theory were, according to MacPherson, developed independently.

Jonathan Fine and Prabhakar Rao [30] used the last corollary to determine the rational intersection homology groups of a complex projective variety $Y$ with an isolated singularity in terms of any desingularization $X$ and its exceptional locus $E$. They proved that, for all $i$,

$$
B_{i}(E)=B_{i}\left(E^{1}\right)-B_{i}\left(E^{2}\right)+B_{i}\left(E^{3}\right)-\cdots \text { where } E^{j}:=\coprod\left(E_{k_{1}} \cap \cdots \cap E_{k_{j}}\right),
$$

In the case that $E$ is a divisor with normal crossings, they went on, by using mixed Hodge theory, to prove a formula for the Betti number $B_{i}(E):=\operatorname{dim} H^{i}(E)$ when $i \geq \operatorname{dim}(X)$ :

$$
B_{i}(E)=B_{i}\left(E^{1}\right)-B_{i}\left(E^{2}\right)+B_{i}\left(E^{3}\right)-\cdots \text { where } E^{j}:=\coprod\left(E_{k_{1}} \cap \cdots \cap E_{k_{j}}\right),
$$

where the $E_{k}$ are the irreducible components of $E$. Combined, those two results provide a lovely "inclusion-exclusion" formula for the intersection homology Betti numbers of $Y$ in the upper half dimensions. The remaining Betti numbers may be determined by using duality.

Walter Borho and MacPherson in $[8, \S 1]$ introduced and studied an important case in which the decomposition of the decomposition theorem is, in fact, canonical. They call a proper map of varieties $f: X \rightarrow Y$ semi-small if for all $r$

$$
\operatorname{codim}\left\{y \in Y \mid \operatorname{dim}\left(f^{-1} y\right) \geq r\right\} \geq 2 r .
$$

(Recall from $\S 3$ that $f$ is said to be a 'small resolution' if the second inequality is strict and if $X$ is smooth.)

Borho and MacPherson, moreover, weakened the hypothesis in the above corollary on $X$ : it does not have to be smooth, but only a rational homology manifold; that is, for all $x \in X$,

$$
H_{r}(X, X-x)= \begin{cases}\mathbb{Q}_{l}, & \text { if } r=2 \operatorname{dim}(X) ; \\ 0, & \text { otherwise. }\end{cases}
$$

It is equivalent, they observe, that $\mathbf{I C} \cdot(X)=\mathbb{Q}_{l, X}$. In this connection, their main result is the following theorem.

Theorem [8, §1]. Let $f: X \rightarrow Y$ be a semi-small proper map of varieties of the same dimension, with $X$ a rational homology manifold. Then $\mathbf{R} f_{*} \mathbb{Q}_{l, X}$ is a perverse sheaf and, in its decomposition into direct summands, $\mathbf{I C} \cdot\left(\bar{V}_{i}, \mathbf{L}_{\mathbf{i}}\right)\left[-e_{i}\right]$, necessarily $e_{i}=\operatorname{codim}\left(V_{i}\right)$; that is, the summands are perverse sheaves too. Moreover, the decomposition into isotypical components - the direct sums of all the isomorphic summands - is canonical and, if $f$ is birational, then one of the isotypical components is $\mathbf{I C} \cdot(Y)$.

Indeed, $\operatorname{codim}\left(\operatorname{Supp}\left(\mathbf{H}^{r}\left(\mathbf{R} f_{*} \mathbb{Q}_{l, X}\right)\right)\right) \geq r$ for $r \geq 0$ because the map is semismall, and $\mathbf{R} f_{*} \mathbb{Q}_{l, X}$ is self-dual because $\mathbb{Q}_{l, X}=\mathbf{I C} \cdot(X)$. Hence, $\mathbf{R} f_{*} \mathbb{Q}_{l, X}$ is 
perverse. Hence, so are its direct summands. Since the category of perverse sheaves is abelian, the isotypical decomposition is canonical. Finally, the last assertion is easy to check.

Borho and MacPherson applied the above theorem (or rather the version of it with $\mathbb{Q}_{X}$ in place of $\mathbb{Q}_{l, X}$ ) to the (semi-small) Springer resolution $\pi: N^{\prime} \rightarrow N$ of the nilpotent cone $N$ in the dual $\mathfrak{g}^{*}$ of the Lie algebra $\mathfrak{g}$ of a connected reductive algebraic group $G$. They also considered Grothendieck's map $\phi: Y \rightarrow \mathfrak{g}^{*}$, which extends $\pi$, and they studied the monodromy action of the fundamental group of the open subset of $\mathfrak{g}^{*}$ of regular semisimple elements (the diagonalizable elements with distinct eigenvalues), recovering Lusztig's construction of Springer's action of the Weyl group $W$, which is a quotient of the fundamental group, on the fibers $H^{*}\left(N_{\xi}^{\prime}, \mathbb{Q}\right)$ of $\mathbf{R} \pi_{*} \mathbb{Q}_{N^{\prime}}$. Their main result is the following theorem.

Theorem [7], [8, §2], [94, 4.8, 4.9]. (1) The nilpotent cone $N$ is a rational homology manifold.

(2) There exists a canonical $W$-stable isotypical decomposition

$$
\mathbf{R} \pi_{*} \mathbb{Q}_{N^{\prime}}=\sum_{(\alpha, \phi)} \mathbf{I C} \cdot\left(\bar{N}_{\alpha}, \mathbf{L}_{\phi}\right)\left[-\operatorname{codim}\left(N_{\alpha}\right)\right] \otimes V_{(\alpha, \phi)}
$$

where the $N_{\alpha}$ are the orbits of $G$ on $N$, the $\mathbf{L}_{\phi}$ are all the various locally constant sheaves of 1 -dimensional $\mathbb{Q}$-vector spaces on $N_{\alpha}$ (they are associated to the various irreducible rational characters of the fundamental group of $\left.N_{\alpha}\right)$, and $V_{(\alpha, \phi)}$ is $a \mathbb{Q}$-vector space of dimension equal to the multiplicity of $\phi$ in the locally constant sheaf $\left(\mathbf{R}^{2 \operatorname{dim}\left(N_{\alpha}\right)} \pi_{*} \mathbb{Q}_{N^{\prime}}\right) \mid N_{\alpha}$.

(3) The group ring of $W$ is equal to the endomorphism ring of $\mathbf{R} \pi_{*} \mathbb{Q}_{N^{\prime}}$ in the category of perverse sheaves. The action of $W$ on the $(\alpha, \phi)$-component is of the form $1 \otimes \rho_{(\alpha, \phi)}$, where $\rho_{(\alpha, \phi)}$ is an absolutely irreducible representation of $W$ on $V_{(\alpha, \phi)}$, and every irreducible complex representation of $W$ is obtained in this way.

In fact, Borho and MacPherson obtain more general results involving parabolic subgroups. In the special case of the general linear group, they obtain a new proof of Lusztig's results on the Green polynomials and the Kostka-Foulkes polynomials.

Assertion (2) above was conjectured by Lusztig [71, §3, Conj. 2] after he established the case of the general linear group. The paper was written and available as a preprint in 1980 .

Assertion (1) has a curious history. Lusztig recalls discussing it with Deligne in 1974. Lusztig gave a lecture at the IHES in which he mentioned some results in representation theory due to Robert Steinberg. Deligne observed that those results would be explained if (1) holds and, the next day, he had a proof. Seven years later in $[71, \S 3$, Rem. (a)], Lusztig stated (1), calling it "an unpublished theorem of Deligne" but saying nothing there about how or when Deligne proved 
it. By the spring of 1981, Borho and MacPherson had proved (2) and (3) in full generality and proved (1) for the general linear group; moreover, using (2) they had reduced (1) to the following lemma, which they conjectured: the trivial representation 1 occurs in the Springer representation on $H^{i}\left(N_{\xi}^{\prime}, \mathbb{Q}\right)$ with multiplicity 1 if $i=0$ and 0 otherwise.

Borho and MacPherson announced Assertions (2) and (3) in [7] but, according to MacPherson, they chose not to discuss (1) in order to keep that Comptes Rendus note sufficiently short. He clearly remembers traveling around Europe, however, lecturing on all three assertions, and asking if (1) was not known. Deligne, at that time, found (1) surprising! At Luminy in July 1981, Borho and MacPherson discussed the lemma with Lusztig. He knew a proof, and so in [7, 2.3] they attribute the lemma to him. Lusztig also told them that Deligne had proved (1). Moreover, Lusztig recalls that he had, in fact, proved the lemma as part of his own (unpublished) proof of (1); that proof involved some known properties of the Green polynomials instead of (2). However, since Deligne had no memory whatsoever of having proved (1) and since they did not realize that Lusztig had his own proof, Borho and MacPherson could feel perfectly comfortable about saying proudly at the beginning of $[7,2.3]$ that (1) "could have been stated in 1930 , but seems to be new."

\section{OTHER WORK AND OPEN PROBLEMS}

A lot of work has been done on the remarkable relation between $L^{2}$-cohomology theory and Hodge theory on the one hand and intersection homology theory on the other. It all began in the winter of 1975-1976 at the State University of New York, Stony Brook, when Cheeger independently found a cohomology theory satisfying Poincaré duality for essentially the same class of spaces as Goresky and MacPherson had considered. Cheeger considered a closed oriented triangulated pseudomanifold $X$. Such an $X$ carries natural piecewise flat metrics. Cheeger formed the $L^{2}$-cohomology groups of the incomplete Riemannian manifold $U$ obtained by discarding all the simplices of codimension 2 or more; those are the cohomology groups $H_{(2)}^{i}(U)$ of the complex of real differential forms $\omega$ on $U$ such that

$$
\int_{U} \omega \wedge * \omega<\infty \text { and } \int_{U} d \omega \wedge * d \omega<\infty
$$

Cheeger found that Poincaré duality could be verified directly or derived formally, in essentially the same way as in the smooth case, from the action of the *-operator on the harmonic forms of the associated Hodge theory-in fact, the full Hodge theory holds - given an inductively defined vanishing condition on the middle dimensional $L^{2}$-cohomology groups of the links, or given a certain more general '*-invariant ideal boundary condition' on the forms. The vanishing condition was later seen to hold whenever $X$ has a stratification by strata of 
even codimension. The theory automatically also works if $X$ is equipped with any metric that on $U$ is quasi-isomorphic to the previous one; then $X$ is said to have 'conical' or 'conelike' singularities. The theory is invariant under smooth subdivision and, more generally, piecewise smooth equivalence.

In the summer of 1976 at Stony Brook, Cheeger informed Sullivan of his discovery. Cheeger was amazed at Sullivan's response: "You know, Goresky and MacPherson have something like that." Sullivan went on to describe the ideas behind their theory. He suggested that Cheeger had found a deRham-Hodge theory dual to their combinatorial one for the middle perversity, and Cheeger later proved it. So, in particular, Cheeger's $L^{2}$-groups are in fact topological invariants. Sullivan also observed that Cheeger's 'ideal boundary condition' corresponds to the central condition in Morgan's (unpublished) extension of their theory to a more general class of spaces. Sullivan proposed that Cheeger and MacPherson talk. Within a few weeks, MacPherson, who was on his way to Paris, passed through Stony Brook to talk to Sullivan. MacPherson talked to Cheeger as well, and was rather surprised to hear about Cheeger's discovery, but agreed that they must be talking about equivalent theories. MacPherson was particularly surprised to hear that there was an $L^{2}$-proof of the Künneth formula, because the product of two middle-allowable cycles is seldom middle allowable.

Cheeger's discovery was an extraordinary byproduct of his work on his proof [18], [20] of the Ray-Singer conjecture, which asserts that on a compact Riemannian manifold the analytic torsion and Reidemeister torsion are equal. In an initial attempt to prove it, Cheeger examined the behavior of the spectrum and eigenfunctions of the Laplacian on differential forms on the level surfaces of a Morse function in a neighborhood of a critical value corresponding to a nondegenerate critical point; that level surface has a 'conical' singularity. Engrossed in writing up his proof of the conjecture until October 1977 and, until February 1978, in obtaining local analytic and combinatorial formulas for the signature and total $L$-class of a pseudomanifold [22], Cheeger did not circulate an announcement of his discovery until the spring of 1978; abridged, it was published in 1979 as [19]. All the details eventually appeared in [21] and [22]. In addition to the first proof of the Künneth formula and the only known explicit local formulas for the $L$ class, Cheeger's analytic methods in intersection homology theory have yielded a vanishing theorem for the intersection homology groups of a pseudomanifold of positive curvature in the pl-sense [21, pp. 139-40], [23]. Moreover, the general methods themselves have also had significant applications to other theories, including index theory for families of Dirac operators [4], the theory surrounding Witten's global anomaly formula [24], and diffraction theory [26].

In the summer of 1977 in the Cheeger dining room about three miles from the Stony Brook campus, Cheeger and MacPherson talked again. This time they considered not the conical metric of a triangulation but the Kähler metric of a 
complex projective variety $X$ with nonsingular part $U$. They conjectured that (i) the $L^{2}$-cohomology group $H_{(2)}^{i}(U)$ is always dual to the intersection homology group $I H_{i}(X)$ and (ii) the pairing is given by integration. In addition, they conjectured that the various standard consequences of Hodge theory-including the Hodge structure, the primitive decomposition, the hard Lefschetz theorem, and the Hodge index theorem-are valid. Those conjectures were published in [21, §7].

With Goresky's help, the preceding conjectures were developed further and discussed in the joint article [25]. There they observed that, to establish the duality conjecture (i), it suffices to prove that the direct image of the presheaf on $U$ formed of the appropriate $L^{2}$-forms of degree $i$ has a 'fine' associated sheaf and that, as $i$ varies, those associated sheaves form a (deRham) complex that satisfies the axioms that characterize IC $(X)$; the cohomology groups of the complex are equal to its hypercohomology groups because the sheaves are fine. They conjectured that each class contains a unique harmonic (closed and co-closed) representative and that splitting the harmonic forms into their $(p, q)$-pieces yields a (pure) Hodge decomposition, compatible with Deligne's mixed Hodge structure on the ordinary cohomology groups of $X$. They noted that the Hodge decomposition would exist if the metric on $U$ were complete, and they suggested that another approach to constructing a Hodge decomposition of $I H .(X)$ is to construct a complete (Kähler) metric. Moreover, they gave a lot of evidence for the validity of the conjectures. This work of Cheeger, Goresky, and MacPherson has lead to a great deal of work by many people.

Zucker was aware of the work of Cheeger, Goresky, and MacPherson that appears in [21] and [40] when he made the following celebrated conjecture, which first appeared in a 1980 preprint of [99]: if $X$ is the Baily-Borel compactification of the quotient space $U$ of a Hermitian symmetric domain modulo a proper action of an arithmetic group $\Gamma$ and if $U$ is provided with the natural complete metric, then the $L^{2}$-cohomology groups are dual to the (middle) intersection homology groups; the forms may take values in a local system on $U$ of a certain type, and then the intersection homology groups are the hypercohomology groups of the DGM extension of the system. Zucker was led to this conjecture by some examples that he worked out $[99, \S 6]$ of his general results [99, (3.20) and (5.6)] about the $L^{2}$-cohomology groups of an arithmetic quotient of a symmetric space. In the examples, the compactification is obtained by adjoining a finite number of isolated singular points, and Zucker was struck by the values of the local $L^{2}$ cohomology groups at these points: they are equal to the singular cohomology groups of the link in the bottom half dimensions and to 0 in the middle and in the top half dimensions. Zucker's work on [99] developed out of an attempt to generalize $\S 12$ of [98]. In [99], the $L^{2}$-cohomology groups were the objects of initial interest; if they are dual to the intersection homology groups, then they are topological invariants. 
Between 1980 and 1987, Zucker's conjecture was proved in various special cases by Zucker himself, by Armand Borel, and by Borel and William Casselman. Finally, in 1987, the general case was proved by Eduard Looijenga [70] and by Leslie Saper and M. Stern [88], [89]. Looijenga uses Mumford's (1975) desingularization of $X$ and the decomposition theorem. Saper and Stern use a more direct method, which they feel will also yield a generalization of the conjecture due to Borel, in which $U$ is an 'equal rank' symmetric space and $X$ is a Satake compactification all of whose real boundary components are equal rank symmetric spaces.

One reason for the great interest in Zucker's conjecture is that it makes it possible to extend the "Langlands program" to cover the important noncompact case, as Zucker indicates in [100]. The program is aimed at relating the $L$ functions of a Shimura variety, which is a 'model' $U_{0}$ of $U$ over a number field, to the automorphic forms associated to the arithmetic group $\Gamma$. The forms are directly related to the $L^{2}$-cohomology groups. The intersection homology groups, constructed using the étale topology, are compatible with the passage modulo a suitable prime of the number field to positive characteristic, where, it is hoped, the $L$-functions may be studied; in this connection, also see Kirwan's discussion [62, pp. 396-98]. In the case of Hilbert modular (or Hilbert-Blumenthal) varieties, Brylinski and Labesse [16] did successfully treat the $L$-functions using intersection homology theory.

The conjectures of Cheeger, Goresky, and MacPherson were also treated with some success in the case that $U$ is the smooth part of a complex projective variety $X$ with isolated singularities. Wu-Chung Hsiang and Vishwambhar Pati [50] gave a proof that $H_{(2)}^{i}(U)$ is dual to $I H_{i}(X)$ if $X$ is a normal surface endowed with the induced (Fubini-Study) metric. Saper [86], [87], who was inspired by the case of the Zucker conjecture, constructed a complete Kähler metric on $U$ whose $L^{2}$-cohomology groups are dual to the intersection homology groups of $X$. Zucker [101] proved that the corresponding Hodge decomposition is compatible with Deligne's mixed Hodge structure, which, in fact, was proved to be pure by J. H. M. Steenbrink [96], who implicitly used the decomposition theorem, and then by Vicente Navarro Aznar [83], who avoided it. Zucker [101, Rem.x(ii), p. 614] notes that the result holds in addition for a Hilbert modular surface, the proof being essentially the same, and that more knowledge about the resolution of the singularities of a Hilbert modular variety of higher dimension will yield the result in the same way in that case as well.

There is other work in the same vein. First, in 1981, Brylinski $[10, \S 3]$ made the following conjecture: if $X$ is embedded in a smooth variety $Y$, say with codimension $c$, and if the regular holonomic $\mathcal{D}$-module $\mathcal{N}$ such that $\mathbf{d e R}(\mathcal{M})=\mathbf{I C} \cdot(X)[c]$ is given the global filtration of Kashiwara and Kawai, then the associated filtration on $\operatorname{deR}(\mathcal{M})$ induces the desired Hodge structure on $I H .(X)$. Second, in a 1985 preprint of [64], János Kollár considered a surjective map $f: X \rightarrow Y$ between projective varieties with $X$ smooth, and he related the sheaves $R^{i} f_{*} \omega_{X}$ to 
certain DGM extensions; then he conjectured a general framework for his results in terms of a corresponding Hodge structure. Third, as mentioned in $\S 7$, in July 1983 Saito [84] announced a theory of 'polarizable Hodge modules' analogous to the theory of pure perverse sheaves, and in [85] he provided the details. Zucker's pioneering work [98], which Deligne had in mind when he came up with his pushforth-and-truncate formula, is now perceived as a cornerstone of Saito's theory. Finally, in 1985, Eduardo Cattani, Aroldo Kaplan, and Wilfred Schmid [17] and, independently, Kashiwara and Kawai [55] generalized that work of Zucker's to higher dimensions: they proved that the intersection homology groups of a smooth variety $X$ are dual to the $L^{2}$-cohomology groups of the complement $U$ in $X$ of a divisor with normal crossings, with coefficients in a local system underlying a polarizable variation of Hodge structure.

Another major topic of research has been the theory of "canonical transforms" of perverse sheaves $\mathbf{S}$; see Luc Illusie's report [52]. The transform $\mathbf{T}(\mathbf{S})$ on $Y$ of $\mathbf{S}$ on $X$ is defined as $\mathbf{R} q_{*}\left(\mathbf{L} \otimes \mathbf{R} p^{*} \mathbf{S}\right)$ where $q: Z \rightarrow Y$ and $p: Z \rightarrow X$ are maps and $\mathbf{L}$ is a local system of rank 1 on $Z$. If $X$ is a vector bundle, $Y$ the dual bundle, and $Z$ their product, then $\mathbf{T}(\mathbf{S})$ is called the vector Fourier transform. If $Y$ is a compact parameter space of a family of subvarieties of $X$ and if $Z$ is the total space (or incidence correspondence), then $\mathbf{T}(\mathbf{S})$ is called the Radon transform. The fundamental theory was developed by Brylinski in a 1982 preprint of [12] on the basis of work of Deligne, of Ryoshi Hotta and Kashiwara, of Gérard Laumon, and of Malgrange. Brylinski also applied the theory to the estimation of trigonometric sums, recovering and extending work of Laumon and Nicholas Katz, and to the study of Springer's representation of the Weyl group via Kashiwara's approach, recovering and extending the results of Springer, of Lusztig, and of Borho and MacPherson.

The transform was used by Laumon [66] to study Langlands' conjecture that there exists a correspondence between the $l$-adic representations of rank $n$ of the Galois group of the algebraic closure of a finite field and the automorphic forms which are eigenvectors of the Hecke operators on $G L_{n}(A)$ where $A$ is the ring of adeles. Ivan Mirković and Vilonen [81] used a Radon transformation, which is like the horocycle transform of Gelfand and Graev (1959), to prove the following conjecture of Laumon and Lusztig: let $G$ be a reductive group, $\mathbf{S}$ a G-equivariant irreducible perverse sheaf, $U$ a maximal unipotent subgroup, and $N$ the nilpotent cone in the dual of the Lie algebra; then (1) in characteristic zero, $\mathbf{S}$ is a character sheaf if and only if its characteristic variety lies in $G \times N$, and (2) in arbitrary characteristic, $\mathbf{S}$ is a tame character sheaf if and only if the direct image of $\mathbf{S}$ on $G / U$ is constructible with respect to the Bruhat cells and is tame. Character sheaves are certain interesting perverse sheaves, which were introduced by Lusztig and studied by him, see [74], and by others as a new way of treating characteristic zero representations of Chevalley groups. 
One important open problem is to determine which maps $f: X \rightarrow Y$ have a natural associated pair of adjoint maps $f_{*}$ and $f^{*}$ on the intersection homology groups. For example, the semi-small resolutions do; see $\S 7$. Another important example is the class of placid maps, which was introduced by Goresky and MacPherson in [44] and [42, §4]. By definition, $f: X \rightarrow Y$ is placid if there exists a stratification of $Y$ such that each stratum $S$ satisfies $\operatorname{codim}\left(f^{-1} S\right) \geq \operatorname{codim}(S)$ (whence equality holds if the map is algebraic). If so, then a map of complexes $f^{*}: \mathbf{I C} \cdot(Y) \rightarrow \mathbf{I C} \cdot(X)$ may be defined using generic geometric chains or using Deligne's construction. Virtually every normally nonsingular map is placid; those maps were considered earlier in Goresky and MacPherson's paper [42, 5.4] and in Fulton and MacPherson's memoir [34], but they were, in fact, introduced and popularized by MacPherson in many lectures at Brown during the years 19751980. To be sure, not every map has such an adjoint pair. An interesting example was given by Goresky and MacPherson in $[41, \S \mathrm{C}]$ : it is the blowing-up $f: X \rightarrow Y$ of the cone $Y$ over a smooth quadric surface in $\mathbf{P}^{3}$; there exist two small resolutions $g_{i}: Y_{i} \rightarrow Y(i=1,2)$ and placid maps $f_{i}: X \rightarrow Y_{i}$ such that $f=g_{i} f_{i}$ but $f_{1}^{*} g_{1}^{*} \neq f_{2}^{*} g_{2}^{*}$.

A related open problem is to determine which subvarieties $X$ of a variety $Y$ have natural fundamental classes in $I H .(Y)$. Not all do. Indeed, if the graph of a map $f: X \rightarrow Y$ between compact varieties has a natural fundamental class in $I H .(X \times Y)$, then that class will define a map $f^{*}: I H .(X) \rightarrow I H .(Y)$, because by the Künneth formula and Poincaré duality,

$$
I H .(X \times Y)=I H .(X) \otimes I H .(Y)=I H .(X)^{\sim} \otimes I H .(Y)=\operatorname{Hom}(I H .(X), I H .(Y)) .
$$

Nevertheless, it might be that there is a well-defined subspace $A .(X)$ of $I H .(Y)$ that is spanned by all reasonable (though not uniquely determined) fundamental classes. It should contain the duals of the Chern classes in the ordinary cohomology groups of all the algebraic vector bundles on $Y$, and it should map onto the space of algebraic cycles in the ordinary homology groups. Given any desingularization $Y^{\prime}$ of $Y$ and embedding of $I H .(Y)$ in $H .\left(Y^{\prime}\right)$ coming from the decomposition theorem, $A .(Y)$ should be the trace of $A$. $\left(Y^{\prime}\right)$. Moreover, the intersection pairing on $I H .(Y)$ should restrict to a nonsingular pairing on $A$. $(Y)$. That nonsingularity is unknown even when $Y$ is nonsingular, and in that case it is one of Grothendieck's 'standard conjectures' [48].

The graph of a placid self-map $f: X \rightarrow X$ is not usually allowable as a cycle for the (middle) intersection homology group; indeed, not even the diagonal itself is. Nevertheless, Goresky and MacPherson [44], [46] proved that these subvarieties carry fundamental classes whose intersection number is equal to the Lefschetz number,

$$
I L(f):=\sum_{i}(-1)^{i} \operatorname{trace}\left(f_{*} \mid I H_{i}(X)\right)
$$


in other words, the Lefschetz fixed-point formula holds for $f$. They also observed that the formula holds when $f$ is replaced by a placid self-correspondence, a subvariety $C$ of $X \times X$ such that both projections $C \rightarrow X$ are placid.

The intersection homology groups with integer coefficients of a complex variety do not usually satisfy Poincaré duality. Goresky and Paul Siegel [47] discovered a 'peripheral group', which measures the failure. Remarkably, this group itself admits a nondegenerate linking pairing, and the Witt class of the pairing is a cobordism invariant. According to Goresky and MacPherson, Sylvan Capell and Julius Shaneson are currently (1988) using the invariant to further knot theory.

Finally, there is the problem of developing a reasonable theory of characteristic numbers for singular varieties. Intersection homology theory yields an Euler characteristic and a signature. It also makes it reasonable to expect that every characteristic number will be the same for a variety $X$ and for any small resolution of $X$. So far, all attempts to lift Chern classes and Whitney classes from ordinary homology groups to intersection homology groups have failed; indeed, Verdier and Goresky gave counterexamples, which were mentioned by Goresky and MacPherson in [46, $\S \mathrm{A}]$ and explained in detail by Brasselet and Gerardo Gonzales-Sprinberg [9]. On the other hand, Goresky [37] has generalized the theory of Steenrod squares from ordinary cohomology theory to intersection homology theory. While Goresky's theory does not generalize completely, it does make it possible to define in the usual way an intersection homology Wu class whose Steenrod square is equal to the homology Wu class. Thus, while significant progress has been made, more remains to be done on that problemthe very problem that motivated the discovery of intersection homology theory.

\section{REFERENCES}

[1] A. Beilinson, How to glue perverse sheaves, K-theory, arithmetic, and geometry. Seminar, Moscow University, 1984-1986, Lecture Notes in Math. 1289, Springer-Verlag 1987, pp. $42-51$.

[2] A. Beilinson and J. Bernstein, Localization of $\mathfrak{g}$-modules, C. R. Acad. Sci. Paris 292 (1981), 15-18.

[3] A. Beilinson, J. Bernstein and P. Deligne, Faiseaux pervers, in "Analyse et topologie sur les espaces singuliers," Astérisque 100, Soc. Math. France, 1982.

[4] J. M. Bismut and J. Cheeger, Invariants êta et indices des familles pour des varietés à bord, C. R. Acad. Sci. Paris 305(I) (1987), 127-130.

[5] J.-E. Björk, "Rings of Differential Operators", North-Holland Publ. Co., 1979.

[6] A. Borel et al., "Algebraic D-Modules," Perspectives in Math. 2, Academic Press Inc., 1986.

[7] W. Borho and R. MacPherson, Représentations des groupes de Weyl et homologie d'intersection pour les variétés nilpotentes, C. R. Acad. Sci. Paris 292 (1981), 707-710.

[8] W. Borho and R. MacPherson, Partial resolutions of nilpotent varieties, in "Analyse et topologie sur les espaces singuliers," Astérisque 101-102, Soc. Math. France, 1982, pp. $23-74$. 
[9] J.-P. Brasselet and G. Gonzalez-Sprinberg, Sur l'homologie d'intersection et les classes de Chern des variétés singulières (espaces de Thom, examples de J.-L. Verdier et $M$. Goresky. With an appendix by Verdier, in "Géométrie algébrique et applications. II (La Rábida, 1984)," Travaux en Cours, no. 23, exposé 2, J.-M. Aroca, T. Sanches-Giralda, J.-L. Vincente (eds.), Hermann, Paris, 1987, pp. 5-14.

[10] J.-L. Brylinski, Modules holonomes à singularites régulières et filtration de Hodge, Algebraic Geometry - La Rábida, Spain 1981, Lecture Notes in Math. 961, Springer-Verlag 1982, pp. 1-21.

[11] J.-L. Brylinski, (Co)-homologie d'intersection et faisceaux pervers, Séminaire Bourbaki 589 Astérisque 92-93 (1982), 129-158.

[12] J.-L. Brylinski, Transformations canoniques, dualité projective, théorie de Lefschetz, transformations de Fourier et sommes trigonométriques, Astérisque, 140-141 (1986), 3-134.

[13] J.-L. Brylinski, "Equivariant intersection cohomology," Inst. Hautes Études Sci., preprint, 1986.

[14] J.-L. Brylinski and M. Kashiwara, Démonstration de la conjecture de Kazhdan and Lusztig sur les modules de Verma, C. R. Acad. Sci. Paris 291 (1980), 373-376.

[15] J.-L. Brylinski and M. Kashiwara, Kazhdan-Lusztig conjecture and holonomic systems, Invent. Math. 64 (1981), 387-410.

[16] J.-L. Brylinski and J.-P. Labesse, Cohomologie d'intersection et fonctions L de certaines variétès de Shimura, Ann. Sci. École Norm. Sup. 17 (1984), 361-412.

[17] E. Cattani, A. Kaplan, and W. Schmid, $L^{2}$ and intersection cohomologies for a polarizable variation of Hodge structure, Invent. Math. 87 (1987), 217-252.

[18] J. Cheeger, Analytic torsion and Reidemmeister torsion, Proc. Nat. Acad. Sci. 74(7) (1977), 2651-2654.

[19] J. Cheeger, On the spectral geometry of spaces with cone-like singularities, Proc. Nat. Acad. Sci. 76 (1979), 2103-2106.

[20] J. Cheeger, Analytic torsion and the heat equation, Ann. of Math. 109 (1979), 259-322.

[21] J. Cheeger, On the Hodge theory of Riemannian pseudomanifolds, in "Geometry of the Laplace operator," Proc. Sympos. Pure Math. 36, Amer. Math. Soc., Providence, RI. 1980, pp. 91-146.

[22] J. Cheeger, Spectral geometry of singular Riemannian spaces, J. Diff. Geom. 18 (1983), 575-657.

[23] J. Cheeger, A vanishing theorem for piecewise constant curvature spaces, in "Curvature and Topology of Riemannian Manifolds," Proc. (1985), K. Shiohana, T. Sakai, T. Sunada (eds.), Lecture Notes in Math. 1201, Springer-Verlag 1986, pp. 333-340.

[24] J. Cheeger, $\eta$-invariants, the adiabatic approximation and conical singularities, J. Diff. Geom. 26 (1987), 175-221.

[25] J. Cheeger, M. Goresky, and R. MacPherson, $L^{2}$-cohomology and intersection homology of singular algebraic varieties, in "Seminar on differential geometry," S. T. Yau (ed.), Princeton University Press, Princeton, NJ, 1982, pp. 303-340.

[26] J. Cheeger and M. Taylor, On the diffraction of waves by conical singularities I, Comm. Pur. App. Math. XXV (1982), 275-331; II, Comm. Pur. App. Math. XXV (1982), 487529.

[27] P. Deligne, Équations différentielles à points singuliers réguliers, Lecture Notes in Math., 163 Springer-Verlag, 1970.

[28] P. Deligne, La conjecture de Weil. I, Publ. Math. IHES 43 (1974), 273-307. 
[29] P. Deligne, La conjecture de Weil. II, Publ. Math. IHES 52 (1980), 137-252.

[30] J. Fine and P. Rao, "On intersection homology at isolated singularities," Northeastern University, preprint, March 1988.

[31] W. Fulton and R. Lazarsfeld, Positive polynomials for ample vector bundles, Ann. of Math. 118 (1983), 35-60.

[32] W. Fulton and R. MacPherson, Intersecting cycles on an algebraic variety, in "Real and complex singularities," P. Holm (ed.), Proc. Oslo (1976), Sitjhoff \& Noorhoof, 1977, pp. 179-197.

[33] W. Fulton and R. MacPherson, Defining algebraic intersections, in "Algebraic GeometryTromsø, Norway 1977," L. D. Olson (ed.), Lecture Notes in Math., 687, 1978, pp. 1-29.

[34] W. Fulton and R. MacPherson, "Categorical framework for the study of singular spaces," Mem. Amer. Math. Soc. 243, 1981.

[35] S. Gelfand and R. MacPherson, Verma modules and Schubert cells: a dictionary, in Séminaire d'algébre Paul Dubreil and Marie-Paule Malliavin, Lecture Notes in Math. 928, Springer-Verlag 1982, pp. 1-50.

[36] M. Goresky, Whitney Stratified Chains and Cochains, Trans. Amer. Math. Soc. 267 (1981), $175-196$.

[37] M. Goresky, Intersection homology operations, Comment. Math. Helv. 59 (1984), 485-505.

[38] M. Goresky, "Kazhdan-Lusztig Polynomials for Classical Groups," Northeastern University, preprint, 1983.

[39] M. Goresky and R. MacPherson, La dualité de Poincaré pour les espaces singuliers, C. R. Acad. Sci. Paris 184 (1977), 1549-1551.

[40] M. Goresky and R. MacPherson, Intersection homology theory, Topology, 149 (1980), 15592.

[41] M. Goresky and R. MacPherson, On the topology of complex algebraic maps, in "Algebraic Geometry—La Rábida, 1981, "Lecture Notes in Math. 961, Springer-Verlag 1982, pp. 119129 .

[42] M. Goresky and R. MacPherson, Intersection Homology II, Invent. Math. 71 (1983), 77129.

[43] M. Goresky and R. MacPherson Morse Theory and Intersection Homology, in "Analyse et topologie sur les espaces singuliers," Astérisque 101-102, Soc. Math. France, 1982, pp. 135-192.

[44] M. Goresky and R. MacPherson, Lefschetz fixed point theorem and intersection homology, in "Intersection cohomology," A. Borel (ed.), Progress in Math. 50, Birkhäuser Boston Inc. 1984, pp. 215-220.

[45] M. Goresky and R. MacPherson, Problems and bibliography on intersection homology, in "Intersection Cohomology," A. Borel (ed.), Progress in Math. 50, Birkhäuser Boston Inc. 1984, pp. 221-233.

[46] M. Goresky and R. MacPherson, Lefschetz fixed point theorem for intersection homology, Comment. Math. Helv. 60 (1985), 366-391.

[47] M. Goresky and P. Siegel, Linking pairings on singular spaces, Comment. Math. Helv. 58 (1983), 96-110.

[48] A. Grothendieck Standard Conjectures on Algebraic Cycles, in "Algebraic Geometry, Papers Bombaby Colloquium," Oxford Univ. Press 1969, pp. 193-199.

[49] D. Hilbert, MATHEMATICAL PROBLEMS, "translated by Dr. Mary Winston Newson," Bull. Amer. Math. Soc. 50 (1902), 437-479. 
[50] W.-C. Hsiang and V. Pati, $L^{2}$-cohomology of normal algebraic surfaces, I, Invent. Math. 81 (1985), 395-412.

[51] R. Joshua, Vanishing of odd-dimensional intersection cohomology, Math. Z. 195 (1987), $239-253$.

[52] L. Illusie, Deligne's $\ell$-adic Fourier Transform, in "Algebraic Geometry-Bowdoin 1985, "S. Bloch (ed.), Proc. Symp. Pure Math. 46(2), 1987, pp. 151-164.

[53] M. Kashiwara, On the maximally overdetermined systems of linear differential equations. I, Publ. Res. Inst. Math. Sc. Kyoto 10 (1975), 563-579.

[54] M. Kashiwara, Faisceaux constructible et systèmes holonomes d'èquations aux dérivées partielles linéaires à points singuliers réguliers, in Séminaire Goulaouic-Schwartz, 197980, Exp. 19.

[55] M. Kashiwara and T. Kawai, The Poincaré lemma for a variation of polarized Hodge structure, Proc. Japan Acad. Ser. A. Math. Sci. 61 (1985), 164-167.

[56] D. Kazhdan and G. Lusztig, Representations of Coxeter Groups and Hecke Algebras, Invent. Math. 53 (1979), 165-184.

[57] D. Kazhdan and G. Lusztig, Schubert varieties and Poincaré duality, in "Geometry of the Laplace operator," Proc. of Sympos. Pure Math. 36, Amer. Math. Soc., Providence, RI. 1980, pp. 185-203.

[58] D. Kazhdan and G. Lusztig, A topological approach to Springer's representations, Adv. Math. 38 (1980), 222-228.

[59] H. King, Topological invariance of intersection homology without sheaves, Topology 20 (1985), 229-234.

[60] F. Kirwan, Rational intersection cohomology of quotient varieties, Invent. Math. 86 (1986), 471-505.

[61] F. Kirwan, Rational intersection cohomology of quotient varieties. II, Invent. Math. 90 (1987), 153-167.

[62] F. Kirwan, Intersection homology and torus actions, J. Amer. Math. Soc. 1 (1988), 385400.

[63] M. Kline, "Mathematical Thought from Ancient to Modern Times," Oxford Univ. Press, 1972.

[64] J. Kollár, Higher direct images of dualizing sheaves. II, Ann. of Math. 124 (1986), 171-202.

[65] A. Lascoux and M. Schützenberger, Polynômes de Kazhdan-Lusztig pour les Grassmanniennes, Young tableaux and Schur functors in algebra and geometry, Astérisque, 87-88 (1981), 249-266.

[66] G. Laumon, Correspondance de Langlands géométrique pour les corps de fonctions, Duke Math. J. 54 (1987), 309-360.

[67] D. T. Lê, "Morsification of D-modules," , Université Paris 7 and Northeastern University, preprint, January, 1988.

[68] D. T. Lê and Z. Mebkhout, Introduction to linear differential systems, in "Singularities," Proc. of Sympos. Pure Math. 40 Part 2, Amer. Math. Soc., Providence, RI. 1983, pp. 3163.

[69] D. T. Lê and Z. Mebkhout, Variétés caractristique et variétés polaires, C. R. Acad. Sci. Paris 1296 (1983), 129-132.

[70] E. Looijenga, $L^{2}$-cohomology of locally symmetric varieties, Compositio Math. 67 (1988), $3-20$. 
[71] G. Lusztig, Green Polynomials and Singularities of Unipotent Classes, Adv. Math. 142 (1981), 169-178.

[72] G. Lusztig, Singularities, character formulas, and a q-analog of weight multiplicities, Astérisque 101-102, Soc. Math. France, 1982, pp. 208-229.

[73] G. Lusztig, "Characters of Reductive Groups over a Finite Field," Ann. of Math. Study 107, Princeton University Press, 1984.

[74] G. Lusztig, Introduction to Character Sheaves, "Finite Group Representations, Arcata 1986," Proc. Symp. Pure Math. 40, Amer. Math. Soc., Providence, RI, 1987, pp. 165-179.

[75] G. Lusztig and D. Vogan, Singularities of Closures of K-orbits on Flag Manifolds, Invent. Math. 71 (1983), 365-379.

[76] R. MacPherson, Global questions in the topology of singular spaces, plenary address, in "Proc. of the International Congress of Mathematicians, vol. 1" August 16-24, 1983, Warszawa, PWN-Polish Scientific Publishers, Warszawa, and Elsevier Science Publishers B.V., Amsterdam, (1984), 213-236.

[77] R. MacPherson and K. Vilonen, Une construction élèmentaire des faisceaux pervers, C. R. Acad. Sci. Paris 292 (1984), 443-446.

[78] R. MacPherson and K. Vilonen, Elementary construction of perverse sheaves, Invent. Math. 84 (1986), 403-435.

[79] Z. Mebkhout, "Cohomologie locale des espaces analytiques complexes," Thèse de Doctorat d'Etat, Université Paris VII, 1979.

[80] Z. Mebkhout, Théorèms de bidualité locale pour les $\mathcal{D}$-modules holonomes, Ark. Mat. 120 (1982), 111-122.

[81] I. Mirković and K. Vilonen, Characteristic varieties of character sheaves, Invent. Math. 93 (1988), 405-418.

[82] R. Mirollo and K. Vilonen, Bernstein-Gelfand-Gelfand reciprocity on perverse sheaves, Ann. Sci. École Norm. Sup. 120 (1987), 311-324.

[83] V. Navarro Aznar, Sur la théorie de Hodge des variétés algébriques á singularités isolées, in "Systèmes Différentiels et Singularités; A. Galligo," M. Granger, Ph. Maisonobe (eds.), Astérisque 130, Soc. Math. France, 1985, pp. 272-307.

[84] M. Saito, Hodge Structure via Filtered D-modules, Systèmes Différentiels et Singularités; A. Galligo, M. Granger, Ph. Maisonobe (eds.), Astérisque 130, Soc. Math. France, 1985, pp. 342-351.

[85] M. Saito, "Mixed Hodge Modules," Res. Inst. Math. Sc. Kyoto, preprint no. 585, 1987.

[86] L. Saper, $L^{2}$-cohomology and intersection homology of certain algebraic varieties with isolated singularities, Invent. Math. 82 (1985), 207-255.

[87] L. Saper, " $L^{2}$-cohomology of isolated singularities," preprint, November (1985).

[88] L. Saper and M. Stern, $L^{2}$-cohomology of arithmetic varieties, Proc. Nat. Acad. Sci. 84 (1987), 5516-5519.

[89] L. Saper and M. Stern, $L^{2}$-cohomology of arithmetic varieties, Duke University, preprint, February (1988). To appear in Ann. of Math.

[90] SGA 4, Théorie des Topos et Cohomologie Etales des Schémas. Tome 1, exposés I-IV, Lecture Notes in Math. 269, Springer-Verlag, 1972; Tome 2, exposés V-VIII Lecture Notes in Math. 270, Springer-Verlag, 1972; Tome 3, exposés IX-XIX Lecture Notes in Math. 305, Springer-Verlag, 1973.

[91] SGA $4 \frac{1}{2}$, Cohomologie Etale, Lecture Notes in Math. 569, Springer-Verlag, 1977. 
[92] SGA 5, Cohomologie l-adique et Fonctions L, Lecture Notes in Math. 589, SpringerVerlag, 1977.

[93] SGA 7 I, Groupes de Monodromie en Géométrie Algébrique, Lecture Notes in Math. 288, Springer-Verlag,1972; II, Lecture Notes in Math. 340, Springer-Verlag, 1973.

[94] T. Springer, Quelques applications de la cohomologie d'intersection, Séminaire Bourbaki 589, Astérisque 92-93, Soc. Math. France, 1982, pp. 249-274.

[95] R. Stanley, Generalized H-Vectors, Intersection Cohomology of Toric Varieties, and Related Results, in "Commutative algebra and combinatorics (Kyoto, 1985)," Adv. Stud. Pure Math. 11, North-Holland, 1987, pp. 187-213.

[96] J. H. M. Steenbrink, Mixed Hodge structures associated with isolated singularities, in "Singularities," P. Orlik (ed.), Proc. Symp. Pure Math. 40(2), Amer. Math. Soc., Providence, RI. 1983, pp. 513-536.

[97] J.-L. Verdier, Spécialisation de faisceaux et monodromie modérée, in "Analyse et topologie sur les espaces singuliers," Astérisque 101-102, Soc. Math. France, 1982, pp. 135-192.

[98] S. Zucker, Hodge theory with degenerating coefficients: $L_{2}$ cohomology in the Poincaré metric, Ann. of Math. 109 (1979), 415-476.

[99] S. Zucker, $L^{2}$-cohomology of Warped Products and Arithmetic Groups, Invent. Math. 70 (1982), 169-218.

[100] S. Zucker, $L^{2}$-cohomology and intersection homology of locally symmetric varieties, II, Compositio Math. 59 (1986), 339-398.

[101] S. Zucker, The Hodge structure on the intersection homology of varieties with isolated singularities, Duke Math. J. 55 (1987), 603-616.

\section{ENDNOTES}

Preface. - These endnotes correct, complete, and update the author's history [36], which is reprinted just above. For the most part, these endnotes respond to comments made to the author shortly after [36] had gone to press and could no longer be modified. In addition, some material reflects recent discussions with Teresa Monteiro Fernandes, Luc Illusie, Masaki Kashiwara, George Lusztig, Prabhakar Rao, Pierre Schapira, and most especially, Mark Goresky. Furthermore, a preliminary draft of the entire work was sent by the editors to a number of referees, and these referees made many apposite comments, which have been incorporated in the current draft. The editors solicited this project in the first place, and more recently suggested adding the foreword and the marginal numbers to the reprint. The author is very grateful for all this support.

Some strongly worded comments were made to the author in 1989 concerning the treatment of algebraic analysis. Indeed, the treatment was marginal. Yet, algebraic analysis played only a supporting role in the development of intersection homology theory. So when [36] was written, the author decided, for the most part, simply to cite a few secondary sources on basic algebraic analysis, as those sources give further information about the mathematics and its provenance. However, the decision was close, since algebraic analysis did play a major role. Furthermore, 
several points of history should really have been discussed. Therefore, a lot of space below is devoted to algebraic analysis.

These endnotes make virtually no attempt to update the discussions of the several lines of research examined in [36]. And no mention is made of the many lines of research that involve intersection homology and perverse sheaves, but that were begun after [36] was written. Tracing all these lines would be rather interesting and certainly worthwhile, but would be a major undertaking because so much work has been done. Indeed, in an email of 21 January 2006 to the author, Goresky wrote: "There are almost 700 papers currently listed in Math Reviews that deal with intersection homology and perverse sheaves. I am slightly familiar with a number of them, perhaps 200 or so, but this [lot] is a minority of the papers, at best. I was quite surprised by this [situation]."

On the other hand, these endnotes indicate many secondary sources, which, in turn, discuss much of the more recent research on intersection homology, perverse sheaves, and related matters.

Citations are of two sorts. If the reference item is listed in [36], and so correspondingly above, then its key is followed by a ' $\mathbf{D}$ '-for example, [5D]. If the reference is, instead, listed at the end of these endnotes, then its key is simply enclosed in brackets - for example, [36].

These endnotes are organized by subject into enumerated subsections. Each includes in its heading, between parentheses, the page number or numbers on which the subject appears in the reprint above. On those pages, the endnote's number appears in the margin to flag the start of the subject.

Endnote 1 (pp. 2,6). - Clint McCrory wrote a letter to the author on 14 January 1989, in which he elaborated on his role in the discovery of intersection homology. His role began with his Brandeis thesis [42]. It was supervised officially by J. Levine, but its topic had been suggested by Sullivan, who also provided a lot of guidance and encouragement.

In "my thesis," McCrory wrote, "I gave a new geometric interpretation of the failure of [Poincaré] duality in terms of the interaction of cycles with the singularities of the space. I introduced the concept of the 'degrees of freedom' of a homology class in a singular space $X$. . . I showed that if $X$ is stratified by piecewise-linear manifolds, a homology class has at least $q$ degrees of freedom if and only if it is represented by a cycle which intersects each stratum in codimension at least $q$ (Corollary 6, p. 101 of my thesis). This condition was the direct precursor of Goresky and MacPherson's concept of perversity. To prove my result, I proved a general position (transversality) theorem for piecewise-linear stratified spaces (Proposition, p. 98 of my thesis)...."

"I visited Brown for the first time during the 1973-74 academic year," McCrory continued. "Bob MacPherson was very interested in my thesis. We sat down with 
it and went over some of the examples. He encouraged me to apply to Brown. I was hired as a Tamarkin Instructor beginning in the fall of 1974. . . . During the summer of 1974, I discussed my thesis and the problem of intersecting cycles, with Bob and Mark Goresky. They left for IHES that fall, and Bob took a copy of my thesis with him. (He lost it and I mailed him another copy.) Word came back that fall (through Bill Fulton) of their breakthrough - to put conditions on how the homologies as well as the cycles intersect the strata, producing new theories satisfying Poincaré duality! I was sorry I'd missed out."

"During the academic year 1975-76," McCrory added, "they started writing up the details, beginning with Mark's thesis. He found that the technology of stratified spaces was insufficient to do what he wanted, so he was forced to use triangulations. But he and Bob persisted in wanting to write up intersection homology without using triangulations. In the summer of 1976, I reminded Bob about the transversality theorem in my (four-year old) thesis, because I realized it was exactly what they needed. Then they decided to go the piecewise-linear route, and I agreed to publish my transversality theorem." This theorem is the subject of McCrory's note [43], which says that the theorem was proved in his thesis and that it refines some of Akin's work, published in 1969.

McCrory's letter inspired Goresky to email the author on 3 February 1989, and say, "I believe it would be very interesting to have a pre-history of intersection homology [theory] because it was an exciting time. Perhaps Clint should write such a history. He would do a very good job of it. However, it would have to include at least the following works," which Goresky enumerated as follows.

(A) Work on the failure of Poincaré duality:

(1) Zeeman (spectral sequence)

(2) McCrory (thesis and related publications)

(3) Kaup and Barthel (singular duality for complex spaces)

(4) Rourke and Sanderson (block bundles and mock bundles)

(5) Whitney (PNAS paper on geometric cohomology)

(6) Borel-Moore (dual of a complex of sheaves)

(B) Work on characteristic classes of singular spaces:

(1) Stiefel (original formula for Stiefel-Whitney classes)

(2) Cheeger (rediscovery of this formula)

(3) Halperin and Toledo (publication of Cheeger's result)

(4) Sullivan (systematic investigation of whitney classes)

(5) MacPherson (Chern classes for singular varieties)

(6) M. H. Schwartz (Chern classes)

(7) Baum-Fulton-MacPherson (Todd classes)

(8) Hirzebruch (L-classes for manifolds with boundary)

(9) Thom (piecewise-linear invariance of Pontrjagin classes) 
(C) Special nature of complex analytic singularities:

(1) Deligne (mixed Hodge structures)

(2) Zucker (variation of Hodge structures over a singular curve)

(3) Kaup and Barthel (applications of Poincaré duality to singular surfaces)

(4) Hamm, Kaup, and Narasimhan (vanishing theorems for singular complex spaces)

(5) Milnor, Lê (isolated hypersurface singularities)

(D) Related developments in algebraic geometry and algebraic analysis:

(1) Kashiwara-Kawai-Sato (theory of $\mathcal{D}$-modules)

(2) Kashiwara-Mebkhout-Brylinski (solutions of a $\mathcal{D}$-module)

(3) Bernstein-Gelfand-Gelfand (singularities of Schubert varieties and their relation to Verma modules)

(E) Stratification Theory:

(1) Whitney (original papers on stratifications)

(2) Thom

(3) Mather

(4) David Stone (piecewise-linear stratification theory)

In his email, Goresky continued by saying, "In many ways I feel it is this last category which had the most profound influence on our thinking. Thom's theory of stratifications was the first serious attempt to understand singularities in a global way. It was this idea which allowed us to stop thinking about triangulations - in a triangulated space you cannot see any clear distinction between one vertex and the next. For example, suppose a space admits a stratification with only even-codimensional strata. How do you notice this [phenomenon], combinatorially, from a triangulation? It is quite a subtle matter."

Goresky added, "Although our early thinking about intersection homology was very much in the spirit of Clint's thinking, since 1978 this [situation] has changed considerably. It now seems that the importance of intersection homology has more to do with the $\mathcal{D}$-module or the Hodge structure or the representation-theoretic side of things than it does with the piecewise-linear or topological aspects. Thus, I believe that a serious discussion of this early work would be severely criticized if it did not contain a discussion of the developments in these fields as well."

Endnote 2 (p. 3). - In addition to the four survey articles cited, there are now (at least) nine more introductions to intersection homology, perverse sheaves, and related matters. They are listed here simply because of their expository value.

Furthermore, the 1983 seminar proceedings [8] by Borel et al. contains more introductory write-ups than the two, $[\mathbf{4 4 D}]$ and $[\mathbf{4 5 D}]$, cited in $[36]$; the foreword 
to [8] explains that some write-ups treat the piecewise-linear theory, some treat the sheaf-theoretic theory, and one treats Siegel's work [66] on cobordism.

The first post-[36] introduction is Kashiwara and Schapira's 1990 book [34]. Its Chapter X gives a rigorous treatment of perverse sheaves on both real and complex analytic manifolds, and its earlier chapters carefully develop the background material from homological algebra, sheaf theory, and microlocal analysis.

The second introduction is Lusztig's ICM report [38]. It gives a concise survey of the applications of intersection homology theory to representation theory up to 1990. Lusztig himself was involved in most of the work.

Arabia's 2003 preprint [1] devotes fifty pages to the general theory of perverse sheaves on singular locally compact spaces, and devotes the remaining ten pages to a detailed treatment of Borho and MacPherson's work [8D] on the Springer correspondence.

Appendix B of Massey's 2003 monograph [41] gives "without proofs," as is explained in $[41$, p. 2], a nearly forty page "working mathematicians guide to the derived category, perverse sheaves, and vanishing cycles."

Schürmann's 2003 book [63] aims, according to Tamvakis's Math Review MR2031639 (2005f:32053), "to develop in detail the functorial theory of constructible sheaves in topology and apply it to study many different kinds of singular spaces . . . triangulated spaces, complex algebraic or analytic sets, semialgebraic and subanalytic sets, and stratified spaces."

Rietsch's 2004 article [55] aims, as is explained on its first page, to provide a "broadly accessible first introduction to perverse sheaves ... intended more to give the flavor and some orientation without delving too much into technical detail." The article ends "with an application, the intersection-cohomology interpretation of the Kazhdan-Lusztig polynomials."

Dimca's 2004 book [12] shows, according to Jeremías López's Math Review MR2050072 (2005j:55002), "topologists and geometers what perverse sheaves are and what they are good for." The book's back cover adds: "Some fundamental results, for which excellent sources exist, are not proved, but just stated and illustrated."

Kirwan and Woolf's 2006 book [35] is a revised and expanded version of Kirwan's 1988 first edition, whose spirit is, according to the new preface, maintained "as an introductory guide ... rather than a textbook. . . Many results are quoted or presented with only a sketch proof." The books culminates in a discussion of the proof of the Kazhdan-Lusztig conjecture. Furthermore, as a referee of the present history noted, Chapter 4 of Kirwan's first edition "is devoted to a brief introduction to Cheeger's work on $L^{2}$-cohomology." 
De Cataldo and Migliorini's survey [11] aims to introduce all the basic concepts and constructions in the theory of perverse sheaves, and to illustrate them with examples. The survey's high point is its extensive discussion of the decomposition theorem, which examines the various proofs and applications of this important theorem.

In addition, another referee suggested mentioning Banagl's 2002 memoir [2] and forthcoming monograph [3], but provided no description of their contents. According to Stong's Math Review MR2189218 (2006i:57061), the memoir "presents an algebraic framework for extending generalized Poincaré duality and intersection homology to pseudomanifolds $X$ more general than Witt spaces."

Endnote 3 (pp. 6,36). - On 14 February 1989, Bill Pardon wrote the author a letter, calling attention to two of his papers, [51] and [22], which he sent in preprint form. The first, he wrote, gives "a proof of Morgan's characteristic variety theorem, but using intersection homology." The second was coauthored by Goresky, and deals with the problem of developing a reasonable theory of characteristic numbers.

Endnote 4 (pp. 7,8). - In [25], Illusie gave a friendly introduction to Verdier's work on the derived category and duality theory, along with a few historical notes.

Endnote 5 (p. 12). - The conjecture about $P_{y, w}$ was made jointly by Kashdan and Lusztig, but left unpublished, according to an email message of 15 December 2006 from Lusztig to the author.

Endnote 6 (p. 14). - There are many more general introductions to algebraic analysis now than the three cited, including (at least) twelve monographs and four surveys. Again, they are listed here simply because of their expository value.

In chronological order, the first monograph is Kashiwara's 1970 Master's thesis, which, in 1995, was Englished and annotated by D'Angelo and Schneiders as [31]. They observed, in their foreword, that it is not simply of historical interest, but serves "also as an illuminating introduction."

The second monograph is the 1979 Paris-Nord (XIII) preprint of [28] of Kashiwara's 1976-1977 course. Monteiro Fernandes was assigned to write it up. In emails to the author on 1-2 January 2007, she described the course as "masterful" and "stimulating." It attracted a young and bright audience. The course reviewed derived categories, Whitney stratifications, and symplectic geometry. It explained the remarkable algebraic-analytic proprerties of PDEs in the setting of $\mathcal{D}$-module theory, especially of holonomic systems, including the Whitney constructibility of their virtual solutions. It culminated in the index theorem. 
The entire course was filled with crucial examples in representation theory. The preprint enjoyed limited distribution, but not long afterwards, she translated them from French to English, and Brylinski wrote a masterful introduction; the result is [28].

The third monograph is Pham's 1979 book [52]. It contains Pham's notes to an introductory course of his also on the analytic theory, and is supplemented by two articles written by three others on Gauss-Manin systems.

The fourth monograph is Schapira's 1985 book [59]. According to Kantor's Math Review MR0774228 (87k:58251), Schapira "gives a detailed and self-contained exposition of . . . the theory of PDEs with holomorphic coefficients as developed by M. Sato, M. Kashiwara et al. . . . the key role being given to microdifferential operators. . . . The book ends with a proof of" Kashiwara's constructibility theorem for holonomic systems. Appendices provide "background on symplectic geometry, homological algebra, sheaves and $\mathcal{O}_{X}$-modules."

The fifth monograph is Mebkhout's 1989 book [49], which "attempts to give a comprehensive introduction" to both the algebraic and the analytic theory, according to Andronikof in his Math Review MR1008245 (90m:32026). "In all, the book is a clear exposition but is tainted with biased references or no reference at all to contemporary work on the subject or to other expository work."

The sixth monograph is Kashiwara and Schapira's 1990 book [34]. This book is devoted to a detailed microlocal study of sheaves on real and complex manifolds, and $\mathcal{D}$-module's are not discussed until the final chapter. Curiously, the Riemann-Hilbert correspondence is not mentioned anywhere.

The seventh monograph is Malgrange's 1991 book [40]. According to the introduction, there are two objectives: a geometric description of holonomic differential systems in one variable, and a study of the effect on such systems of the Fourier-Laplace transform. Chapter I reviews the basic theory of $\mathcal{D}$-modules; most proofs are omitted, and the rest, sketched.

The eighth monograph is Granger and Maisonobe's 1993 set of notes [23], which offers "a short course presenting the basic results in the theory of analytic D-modules," according to D'Angolo in his Math Review MR1603609 (99c:32008).

The ninth monograph is Björk's 1993 book [7], which offers a comprehensive development of the analytic theory, and includes seven appendices covering background material in algebra, analysis, and geometry. On p. 5, Björk explains that his own book [5D] "was written prior to to the development of regular holomonic modules and is therefore less oriented to the topics of [7]." In his Math Review MR1232191 (95f:32014) of the book, Macarro observes that it "contains detailed proofs of almost all the main results of the theory," but he feels that "the style . . . does not help to distinguish the crucial points from the auxiliary or complementary ones." Furthermore, he observes that "each chapter ends 
with some bibliographical and historical notes," but says that they "are often incomplete," or even incorrect.

The tenth monograph is Schneiders' 1994 introduction [65], which develops the more elementary aspects of the analytic theory.

The eleventh monograph is Coutinho's 1995 book [10]. It is a lucid introduction to the more elementary aspects of the algebraic theory in the important and illustrative special case in which the ambient variety is the affine space.

The twelveth monograph is Kashiwara's 2003 book [32]. According to Marastoni's review MR1943036 (2003i:32018), it "is substantially self-contained and remarkably clear and concise, . . . an excellent reference book on analytic $\mathcal{D}$ modules, microlocal analysis and $b$-functions, and also as a good introduction to these theories."

The four surveys are these: Oda's [50] of 1983, Gelfand and Manin's [18] of 1999, Dimca's [12, Sec. 5.3] of 2004, and Kirwan and Woolf's [35, Ch. 11] of 2006. All four are excellent. None have proofs, although Kirwan and Woolf's does sketch a couple. Moreover, all four give a lot of precise references to the literature, where the proofs are found. Furthermore, Dimca's points out the differences between the analytic approach and the algebraic approach. Oda's, unlike the other three, could have been cited in [36].

Endnote 7 (p. 15). - Schapira wrote a pleasant sketch [62] of Sato's life and mathematics on the occasion of his receipt of the 2002/03 Wolf prize.

Without doubt, the most prominent member of Sato's school is Kashiwara. He has made a number of fundamental contributions to algebraic analysis, many of which are discussed in [36] and in these endnotes.

Kashiwara's contributions began with his Master's thesis, mentioned in the preceding endnote. It was written in Japanese, and submitted to Tokyo University in December 1970. Twenty-five years later, it was published in the annotated English translation [31], which has two forewords. In the second, Schapira observed that Kashiwara's thesis drew inspiration from some "pioneering talks" by Sato and from Quillen's Harvard PhD thesis [53], and that Kashiwara's thesis and Bernstein's papers [4] and [5] are the "seminal" works in algebraic analysis.

Endnote 8 (pp. 16, 18, 20). - A nearly definitive generalization of the RiemannHilbert problem was formulated by Kashiwara and published in 1978 by Ramis [54, p. 287], who called it a conjecture. However, this formulation differs from that given on p. 16 of [36]: notably, Ramis asserted that the functor $\mathcal{M} \mapsto \operatorname{Sol}(\mathcal{N})$ is an equivalence of categories, but not that it is natural in the ambient space, in the sense that it commutes with direct image, inverse image, exterior tensor 
product, and duality. However, this naturality is proved whenever the equivalence is proved; indeed, the naturality is used in an essential way in every proof of the equivalence.

Ramis said he had learned about Kashiwara's formulation in February 1977 from Malgrange. In turn, according to Schapira [60] and [61], Malgrange had learned about it directly from Kashiwara in Stockholm in May 1975.

Mebkhout did not, in fact, fully solve the generalized problem in his 1979 doctoral thesis [79D]. Rather, as he himself explained in his 1980 summary [45] of Chapter V of his thesis, he solved only the analogous problem for differential operators of infinite order. At the same time, he expressed his hope of deducing the solution for operators of finite order. Shortly afterwards, he succeed. He detailed the full solution in [47] and [48]. And he and Lê sketched it nicely in [68D, pp. 51-57].

Meanwhile, Kashiwara found a full solution. He announced it in [54D] in 1980, and detailed it in [29]. His approach is somewhat different. Notably, using the $\bar{\partial}$-operator, he constructed an inverse to the functor $\mathcal{M} \mapsto \operatorname{Sol}(\mathcal{M})$. However, in establishing the naturality, he too used differential operators of infinite order.

Beilinson and Bernstein found a suitable algebraic version of the theory, and Bernstein lectured on it in the spring and summer of 1983. Borel "elaborated" on Bernstein's notes in [6D, Chaps. VI-VIII], according to [6D, p. vii].

Endnote 9 (p. 17). — Set $d:=\operatorname{dim}(X)$. Let $\mathcal{M}$ be a nonzero coherent $\mathcal{D}$-module, and $Y$ a component of its characteristic variety $\mathrm{Ch}(\mathcal{M})$. Then, as asserted,

$$
\operatorname{dim}(Y) \geq d
$$

This important lower bound is sometimes called "Bernstein's inequality" to honor Bernstein's discovery of it in his great 1972 paper [5, Thm.1.3, p. 275]. For example, this designation is used by Björk [5D, p. 9], by Coutinho [10, p. 83, p. 104], by Ehlers [6D, p. 178, p. 183], and by Oda [50, p. 39].

Bernstein came to this bound, according to Bernstein and S. I. Gelfand [6, p. 68], from a question posed by I. M. Gelfand [18, p. 262] at the ICM in 1954: given a real polynomial $P$ on $\mathbb{R}^{n}$ with nonnegative values, and given a $C^{\infty}$ function $f$ on $\mathbb{R}^{n}$, consider the function $\Gamma_{f}$ in the complex variable $\lambda$,

$$
\Gamma_{f}(\lambda):=\int P^{\lambda}(x) f(x) d x
$$

which is analytic for $\Re(\lambda)>0$; can $\Gamma_{f}$ be extended meromorphically to all $\lambda \in \mathbb{C}$ ? Indeed, it can! Proofs were published by Atiyah in 1968 and, independently, by Bernstein and S. I. Gelfand [6] in 1969; both proofs rely on Hironaka's 1964 resolution of singularities. In 1972, Bernstein [5] offered an elementary and elegant new proof, which is presented in detail in [5D, pp. 12-15]; the key is the bound. 
However, Kashiwara had, independently, already established the bound in his 1970 thesis; witness [31, p. 38]. Apparently, this fact was not well known, because Kashiwara's name was not associated with the bound. Ironically, in the introduction to his book [5D, pp. v], Björk wrote: "I have had the opportunity to learn this subject from personal discussions with M. Kashiwara. His thesis contains many of the results in this book." Furthermore, Oda's survey was intended to provide background for Kashiwara's report to an audience in Tokyo.

Kashiwara proved the bound via a fairly elementary induction on $d$. Björk gave two proofs in same spirit in [5D, pp. 9-12]. Bernstein gave a somewhat more sophisticated argument involving the Hilbert polynomial of $Y$. His argument was simplified somewhat by Joseph, and this simplification was presented by Ehlers [6D, p. 178] and by Coutinho [10, p. 83].

Both Kashiwara [31, p. 45] and Bernstein [5, Rmk., p. 285] said that the bound is related to the homological properties of $\mathcal{D}$-modules, but neither went into detail. However, Kashiwara went on to give a simple proof that $\mathcal{D}_{X}$ has finite global homological dimension for any $X$. Bernstein simply cites Roos's paper [56], which had just appeared; in it, Roos proved that $\mathcal{D}_{X}$ has finite weak global homological dimension when $X$ is the affine space.

Björk [5D, pp. $\mathrm{x}-\mathrm{xi}$ ] gave a proof of the bound using this same finiteness theorem of Roos's. Björk combined the latter with another homological formula, which he proved on the basis of some earlier work of Roos's. Björk also used this formula to settle another matter: $\operatorname{dim}(Y)$ is equal to the degree of Bernstein's Hilbert polynomial. The problem is that Bernstein's filtration is not the one used to define the characteristic variety. Ehlers [6D, pp. 183-185] follows Björk's approach here, and indeed quotes some of his results.

Bernstein [5, Rmk., p. 285] also said that the bound "is a simple consequence of the hypothesis [conjecture] on the 'integrability of characteristics' [the involutivity of the characteristic variety] formulated by Guillemin, Quillen, and Sternberg in $[24$, p. 41]" in 1970. They proved it in a special case, and applied it to the classification of Lie algebras. In 1973, Kashiwara, Kawai, and Sato [58, Thm. 5.3.2, p. 453] proved the conjecture in the general complex analytic case; see also Kashiwara's book [28, Cor. 3.1.28]. In 1978, Malgrange [39] gave a new and cleaner proof. All three of those proofs involve analysis on a localization of the cotangent variety, or "microlocalization." In 1981, Gabber [16, Thm. 1, p. 449] proved the purely algebraic version of the original conjecture [24, p. 59] under a mild finiteness hypothesis. In 1990, Kashiwara and Schapira [34, Thm.6.5.4, p. 272] proved a real analytic version of the conjecture, in a way they describe on p. 282 as "radically different" and purely "geometric." The involutivity directly implies the bound, and has been derived in this way in most expositions for the last twenty years; for example, see Coutinho's introduction [10, p. 83]. 
Endnote 10 (p. 17). - Kashiwara's Theorem (3.1) in [53D, p. 563] says essentially that, if $\mathcal{M}$ is a holonomic $\mathcal{D}$-module on $X$, then the sheaves $\operatorname{Ext}_{\mathcal{D}}^{i}\left(\mathcal{M}, \mathcal{O}_{X}\right)$ are constructible with respect to some Whitney stratification. A few years later in $[27, T h m .4 .8]$, he generalized the theorem by replacing $\mathcal{O}_{X}$ by a second holonomic $\mathcal{D}_{X}$-module.

Endnote 11 (p. 17). - Oda described three other definitions of regular singular points in Subsection (4.5) of his survey [50, pp.40-41]. He noted that the four definitions seem unrelated, but are equivalent; in fact, Kashiwara and Kawai devoted their 166-page paper [33] to the proof, which uses other, microlocal characterizations, involving microdifferential operators of finite order and of infinite order, and reduction to special cases treated by Deligne in $[\mathbf{2 7 D}]$.

Endnote 12 (pp. 18,21). - In the statements of the two theorems, only secondary sources are cited, and in his letter [60] to the author, Schapira asked why so. The answer is this: these sources are being credited for their formulations and discussions, not for their discoveries. The context makes this fact clear, but with hindsight, it is also clear that, regrettably, a casual reader might be mislead.

Endnote 13 (p. 19). - Regrettably, what is written might lead some to think, as Schapira suggested in his commentary [61], that, when Brylinski and Kashiwara jointly resolved the Kazhdan-Lusztig conjecture, Brylinski contributed the lion's share.

In fact, as explained in [36], Kashiwara was the only expert among a halfdozen, who recognized the potential in Brylinski's ideas and who was kind enough and interested enough to offer to collaborate with him to make something of them. Brylinski is described as an eager beginner, and Kashiwara, as a generous established expert.

Unfortunately, the author was unable to determine to what extent these ideas had been developed independently by Kashiwara before he received Brylinski's program of proof, and the account in [36] is described only from Brylinski's point of view, as detailed in his letter of 4 October 1988 to the author and approved in an email of 26 October 1988.

Endnote 14 (p. 21). - The bibliographically correct version of Mebkhout's article $[\mathbf{8 0 D}]$ is $[46]$. This "article reproduces Chapter 3 of the author's thesis," according to Schapira's review of it, MR0660129 (84a:58075).

Endnote 15 (p. 21). - Yes, as a referee surmised, these words were said tongue in cheek. 
Endnote 16 (p. 21). - As the context makes clear, in this theorem, the ambient space is an algebraic variety, so of finite type over a field. However, in practice, we are sometimes led to consider nontrivial, but manageable, inductive limits of varieties, as a referee remarked and Goresky seconded. For example, in the geometric Langlands program, we are led to consider affine, or loop, Grassmannians $\mathbf{G}(\mathbb{C}((t))) / \mathbf{G}(\mathbb{C}[[t]])$ that are not finite dimensional, and in the study of Shimura varieties and discrete groups, we are led to consider Borel-Serre partial compactifications of symmetric varieties whose boundary has countably many boundary components. In these case, the category of perverse sheaves is only locally Artinian.

Endnote 17 (p. 21). - In Part (1), the perverse sheaf $\mathbf{S}$ must be on $X$, not its subvariety $V$; otherwise, it would surely be curious to speak of the restriction of $\mathbf{S}$ to $V$. Yet a referee suggested this implicit condition be made explicit. Goresky explained why, in an email to the author on 30 December 2006.

"The reason," Goresky wrote, "is that everything depends on the shift. If you view $\mathbf{L}$ as a sheaf on $X$, then it is not perverse. Rather, $\mathbf{L}[-c]$ is perverse, as a sheaf on $X$. If you view $\mathbf{L}$ as a sheaf on $V$, then it is perverse, while $\mathbf{L}[-c]$ is not a perverse sheaf on $V$. So it is potentially confusing, and adding the words "on $X$ ' will help to keep the reader from becoming confused."

Endnote 18 (pp. 22, 23). - A referee observed that it is common nowadays to omit the upper-case $\mathbf{R}$ from the notation for the perverse sheaves of nearby cycles and of vanishing cycles.

Endnote 19 (pp. 23). - Lê's preprint [67D] finally appeared in print as [37]. The cited, but unreferenced, work of Dubson, of Ginzburg, and of Sabbah appeared in [14], in [19] and [20], and in [57]. Kashiwara proved a more general real version of the intersection formula in [30, Thm. 8.3, p. 205]. Schürmann gave a careful historical survey of the work done up to 2003 on this formula and related formulas, emphasizing the real case, in the introduction to his article [64].

In [69D, p. 130], Lê and Mebkhout used Kashiwara's index theorem, citing Kashiwara's preprint of [28]; for more about the latter work; see Endnote 6. On p.xiii of Brylinski's introduction to the published version [28], Brylinski noted the "beautiful fact" that the topological invariant in Kashiwara's theorem "is nothing else but" MacPherson's local Euler obstruction, a fact he attributed to Dubson, citing Dubson's 1982 Paris thesis and the joint note [9]; Dubson's thesis itself hasn't appeared in print, but see his note [14]. Also on p.xiii, Brylinski explained the connection between Kashiwara's theorem and vanishing cycles. Earlier, in 1973, Kashiwara had announced the theorem in [26]. 
Endnote 20 (p. 26). - By definition [3D, Sect.6.2.4, p. 162], a perverse sheaf is of geometric origin if it can be obtained from the constant sheaf on a point by repeatedly applying Grothendieck's six operations $\left(\mathbf{R} f_{*}, \mathbf{R} f_{!}, \mathbf{R} f^{*}, \mathbf{R} f^{!}, \mathbf{R H o m}\right.$, and $\otimes^{L}$ where $f$ is a morphism of algebraic varieties) and by repeatedly taking simple perverse constituents.

Endnote 21 (p. 28). - A referee pointed out that "the two displayed formulas are identical. The first occurrence needs to be replaced." Very likely, it should be replaced by this formula:

$$
\operatorname{dim} I H_{i}(Y)=\operatorname{dim} H_{i}(X)-\operatorname{dim} H_{i}(E) .
$$

Also, $i$ must be subject to the lower bound $i \geq n$.

Rao helped the author recover the intended formula via an email received on 28 December 2006. He noted that the above formula constitutes Item a) on p. 339 of the published version [15] of the preprint [30D]. He added that the preprint "gave a more down-to-earth proof of Item a) using a result of Goresky-MacPherson. The referee insisted that I replace it with the more opaque proof [directly] using the Decomposition Theorem on page 338."

Endnote 22 (p. 32). - On 4 May 1989, Karl-Heinz Fieseler and Ludger Kaup sent the author a half-dozen reprints of their papers, which appeared between 1985 and 1988. In them, the authors prove a number of theorems of Lefschetz type using purely topological methods, rather than Hodge-theoretic methods.

In fact, as Goresky explained to the author in an email of 7 January 2007, "there are a lot of topological papers concerning Lefschetz-type theorems and intersection homology and perverse sheaves. Schürmann's book [63] contains references to results of Brasselet, Fieseler, Kaup, Hamm, Lê, Goresky, MacPherson, Schürmann and others, and I think the list is probably longer by now."

Endnote 23 (p. 34). - In an email to the author on 15 December 2006, Lusztig clarified the history of the conjecture as follows: "You say that Mirkovic and Vilonen proved a conjecture of Laumon and Lusztig, which has two parts, (1) and (2). In fact, in Part (1), one implication (if $\mathbf{S}$ is a character sheaf, then its characteristic variety is contained in an explicit Lagrangian) was proved by me, and I conjectured to Mirkovic and Vilonen that the converse holds; they proved it. I am not sure about Laumon."

"Part (2)," Lusztig continued, "was not conjectured by Laumon and me, nor proved by Mirkovic and Vilonen. In fact, again, before their paper was written, I proved one implication (namely, any character sheaf has the property stated in (2)). After Vilonen gave me a preprint of their paper, I realized that, on the basis of that preprint, one can deduce the converse of the property in (2). I told him 
so, and they included this deduction in the final version of the paper. So, here, the correct statement is that I proved the converse after their paper was written. I think that that Laumon has nothing to do with (2)."

Endnote 24 (p. 36). - Concerning the failure of Poincaré duality for the intersection homology groups with integer coefficients, a referee asked for clarification of what precisely fails. The following clarification was provided in an email to the author on 30 December 2006 by Goresky.

"For a compact $n$-dimensional manifold $M$," Goresky wrote, "the intersection pairing

induces a mapping

$$
H_{n-i}(M ; \mathbb{Z}) \otimes H_{i}(M ; \mathbb{Z}) \rightarrow \mathbb{Z}
$$

$$
H_{n-i}(M ; \mathbb{Z}) \rightarrow \operatorname{Hom}\left(H_{i}(M ; \mathbb{Z}), \mathbb{Z}\right),
$$

which becomes an isomorphism after tensoring with the rational numbers. But even more is true. Since $H^{i}(M ; \mathbb{Z}) \cong H_{n-i}(M ; \mathbb{Z})$, the universal coefficient theorem says that in fact there is a split short exact sequence

$$
0 \rightarrow \operatorname{Ext}\left(H_{i}(M ; \mathbb{Z}), \mathbb{Z}\right) \rightarrow H_{n-i}(M ; \mathbb{Z}) \rightarrow \operatorname{Hom}\left(H_{i}(M ; \mathbb{Z}), \mathbb{Z}\right) \rightarrow 0,
$$

and this fact is (usually) false for singular varieties, even when $H_{*}$ is replaced by $I H_{*} . "$

"Here," Goresky continued, "is the sheaf theoretic way of saying this: the dualizing sheaf $\mathbf{D}(\mathbb{Z})$ is defined to be $f^{!}(\mathbb{Z})$ where $f: X \rightarrow\{$ point $\}$. The intersection pairing defines a mapping

$$
\mathbf{I C}(\mathbb{Z}) \rightarrow \mathbf{R H o m}(\mathbf{I C}(\mathbb{Z}), \mathbf{D}(\mathbb{Z}))
$$

(with appropriate shifts), where $\mathbf{I C}(\mathbb{Z})$ denotes the complex of intersection chains with integer coefficients. If $X$ is a manifold, then this mapping is a quasiisomorphism. But if $X$ is a singular space, then this mapping only becomes a quasi-isomorphism after tensoring with the rational numbers."

"More generally," Goresky wrote, "the dualizing sheaf $\mathbf{D}(R)$ can be defined for any sufficiently nice ring $R$, and we always get a mapping

$$
\mathbf{I C}(R) \rightarrow \mathbf{R H o m}(\mathbf{I C}(R), \mathbf{D}(R)) .
$$

And if $R$ is a field, then this mapping is a quasi-isomorphism. But if $R$ is not a field, then this mapping is not usually a quasi-isomorphism."

"Paul Siegel and I," Goresky continued, "figured out sufficient conditions for the obstruction to vanish. When I later mentioned these conditions to Pierre Deligne, he indicated, in his usual polite and friendly way, that he already [knew] these facts. (I don't know when he figured them out. He did not include this [material] in Astérisque 100 [ 3D], and he never published anything on the subject. 
It is only one of many wonderful results that Pierre has figured out, but never published.)"

"Finally," Goresky wrote, "I should mention that Poincaré duality over the integers implies that the intersection pairing on the middle degree homology of a $4 k$ dimensional space, will be unimodular. This [statement] is true for $4 k$ dimensional manifolds, but it does not, in general, hold for $4 k$-(real-)dimensional algebraic varieties and intersection homology."

\section{REFERENCES}

[1] Arabia, A., "Correspondance de Springer," (Prépublication) Institut de Mathématique de Jussieu, Univ. Paris 7, obtained (10 Dec 2006) from http://www.institut.math.jussieu. $\mathrm{fr} /$ arabia/math/Pervers.pdf.

[2] M. Banagl, "Extending intersection homology type invariants to non-Witt spaces," Mem. Amer. Math. Soc. 160, 2002.

[3] M. Banagl, "Topological Invariants of Stratified Spaces," Springer Monographs in Math., to appear in January 2007.

[4] Bernstein, J., Modules over a ring of differential operators. An investigation of the fundamental solutions of equations with constant coefficients, Functional Anal. Appl. 5 (1971), 89-101.

[5] Bernstein, J., The analytic continuation of generalized functions with respect to a parameter, Functional Anal. Appl. 6 (1972), 273-285.

[6] Bernstein, J., and Gelfand, S. I., Meromorphic property of the functions $P^{\lambda}$, Functional Anal. Appl. 3 (1969), 68-69,

[7] Björk, J.-E., "Analytic D-modules and applications," Mathematics and its Applications 247, Kluwer Academic Publishers Group, 1993.

[8] Borel et al., "Intersection Cohomology," Progress in Math. 50, Birkhäuser Boston Inc. 1984

[9] Brylinski, J.-L., Dubson, A., and Kashiwara, M., Formule de l'indice pour modules holonomes et obstruction d'Euler locale, C. R. Acad. Sci. Paris S. I Math. 293, no. 12, (1981), 573-576.

[10] Coutinho, S., "A primer of algebraic D-modules," London Mathematical Society Student Texts, 33, Cambridge Univ. Press, 1995.

[11] de Cataldo, M., and Migliorini, L., a survey under preparation for the Bull. Amer. Math. Soc.

[12] Dimca, A., "Sheaves in topology. Universitext," Springer-Verlag, 2004.

[13] Dubson, A. Formule pour l'indice des complexes constructibles et des Modules holonomes, C. R. Acad. Sci. Paris Sér. I Math. 298, no. 6, (1984), 113-116;.

[14] Dubson, A., Formule pour les cycles évanescents, C. R. Acad. Sci. Paris S. I Math. 299, no. 6, (1984), 181-184; Erratum, C. R. Acad. Sci. Paris 299, no. 6, (1984), 193.

[15] Fine, J., and Rao, P., On intersection homology at isolated singularities, Algebras Groups Geom. 5 (1988), no. 4, 329-340.

[16] Gabber, O., "The integrability of the characteristic variety," Amer. J. Math., 103 (1981), no. 3, 445-468.

[17] Gelfand, I. M., "Some aspects of functional analysis and algebra," in "Proceedings of the International Congress of Mathematicians, Amsterdam, 1954," Vol. 1 North-Holland Publishing Co. 1957, pp. 253-276. 
[18] Gelfand, S. I., and Manin, Yu., "Homological algebra," Transl. from the 1989 Russian original by the authors, Reprint of the original English edition from the series Encyclopaedia of Mathematical Sciences, Algebra, V, Encyclopaedia Math. Sci., 38, Springer, 1994; Springer, 1999.

[19] Ginzburg, V., A theorem on the index of differential systems and the geometry of varieties with singularities, Dokl. Akad. Nauk SSSR 281 (1985), no. 3, 521-525; English translation, Soviet Math. Dokl. 31 (1985), no. 2, 309-313.

[20] Ginzburg, V., Characteristic cycles and vanishing cycles, Invent. Math. 84 (1986), 327-402.

[21] Goresky, M., and MacPherson, R., "Stratified Morse theory," Ergebnisse der Mathematik und ihrer Grenzgebiete (3) 14, Springer-Verlag, 1988.

[22] Goresky, M., and Pardon, W., Wu numbers of singular spaces, Topology 28 (1989), no. 3, 325-367.

[23] Granger, M., and Maisonobe, P. A basic course on differential modules. in "Éléments de la théorie des systèmes différentiels. D-modules cohérents et holonomes (Nice, 1990)," Travaux en Cours, 45, Hermann, 1993, pp. 103-168.

[24] Guillemin, V., Quillen, D., and Sternberg, S., The integrability of characteristics, Comm. Pure Appl. Math. 23, no. 1, (1970), 39-77.

[25] Illusie, L., Catégories dérivées et dualité, travaux de J.-L. Verdier, Enseign. Math. (2) 36 (1990), 369-391.

[26] Kashiwara, M., Index theorem for maximally overdetermined systems of linear differential equations. Proc. Japan Acad. 49 (1973), 803-804.

[27] Kashiwara, M., On the holonomic systems of micro-differential equations, II, Invent. Math. 49 (1978), 121-135.

[28] Kashiwara, M., Systems of microdifferential equations, Notes and translation by T. Monteiro Fernandes, introduction by J.-L, Brylinski, Progress Math 34, Birkhäuser, 1983.

[29] Kashiwara, M., The Riemann-Hilbert problem for holonomic systems, Publ. Res. Inst. Math. Sci. 20, no. 2, (1984), 319-365.

[30] Kashiwara, M., Index theorem for constructible sheaves. Astérisque 130 (1985), 193-209.

[31] Kashiwara, M., "Algebraic study of systems of partial differential equations," Mem. Soc. Math. France (N.S.) No. 63, 1995; Erratum, Bull. Soc. Math. France 125 (1997), no. 2, 313.

[32] Kashiwara, M., "D-module and microlocal calculus," translated from the 2000 Japanese original by Mutsumi Saito, Translations of Mathematical Monographs, 217, Iwanami Series in Modern Mathematics, Amer. Math. Soc., 2003.

[33] Kashiwara, M., and Kawai, T., On holonomic systems of microdifferential equations. IIISystems with regular singularities. Publ. Res. Inst. Math. Sci. 17, no. 3, (1981), 813-979.

[34] Kashiwara, M., and Schapira, P., "Sheaves on Manifolds," Springer, 1990; corrected reprint, 1994.

[35] Kirwan, F. and Woolf, J., "An introduction to intersection homology theory," Chapman \& Hall/CRC, 2006.

[36] Kleiman, S., The development of intersection homology theory, in "A century of mathematics in America, Part II," Hist. Math., 2, Amer. Math. Soc., 1989, pp. 543-585, reprinted here as Sections 1-8 and the first list of references.

[37] Lê, D. T. Morsification of D-modules, Bol. Soc. Mat. Mex., III. Ser. 4, No.2, (1998), 229248. 
[38] Lusztig, G., Intersection cohomology methods in representation theory, Proceedings of the International Congress of Mathematicians, Vol. I, II (Kyoto, 1990), Math. Soc. Japan, 1991, pp., 155-174.

[39] Malgrange, B., "L'involutivité des caractéristiques des systèmes différentiels et microdifférentiels," Séminaire Bourbaki, 30e année (1977/78), Exp. No. 522, Lecture Notes in Math., 710, Springer,1979, pp. 277-289.

[40] Malgrange, B., "Équations difféntielles à coefficients polynomiaux," Progress Math., 96. Birkhser Boston, Inc., 1991.

[41] Massey, David B., "Numerical control over complex analytic singularities." Mem. Amer. Math. Soc., vol. 163, no. 778, 2003.

[42] McCrory, C., "Poincaré duality in spaces with singularities," Brandeis PhD thesis, May 1972.

[43] McCrory, C., Stratified general position, in "Algebraic and geometric topology," Proc. Sympos., Univ. California, Santa Barbara, Calif., 1977, Lecture Notes in Math., 664, SpringerVerlag, 1978, pp. 142-146.

[44] Mebkhout, Z. Théorème de dualité pour les $D_{x}$-modules cohérents, C. R. Acad. Sci. Paris Sér. A-B 285, no. 12, (1977), A785-A787.

[45] Mebkhout, Z., Sur le problème de Hilbert-Riemann, C. R. Acad. Sci. Paris Sér. A-B 290, no. 9, (1980), A415-A417.

[46] Mebkhout, Z., Théorèmes de bidualité locale pour les $\mathcal{D}_{X}$-modules holonomes, Ark. Mat. 20, no. 1, (1982), 111-124.

[47] Mebkhout, Z. Une équivalence de catégories, Compositio Math. 51, no. 1, (1984), 51-62.

[48] Mebkhout, Z. Une autre équivalence de catégories, Compositio Math. 51, no. 1, (1984), 63-88.

[49] Mebkhout, Z. Le formalisme des six opérations de Grothendieck pour les $\mathcal{D}$-modules cohérents. With supplementary material by the author and L. Narvz Macarro. Travaux en Cours 35, Hermann, 1989.

[50] Oda, T., Introduction to algebraic analysis on complex manifolds, in "Algebraic varieties and analytic varieties (Tokyo, 1981)," S. Iitaka (ed.), Adv. Stud. Pure Math., 1, NorthHolland, Amsterdam, 1983, pp. 29-48.

[51] Pardon, W., Intersection homology Poincaré spaces and the characteristic variety theorem, Comment. Math. Helv, 65 (1990), 198-233.

[52] Pham, F., "Singularités des Systèmes Différentiels de Gauss-Manin." with contributions by Lo Kam Chan, Philippe Maisonobe and Jean-ienne Rombaldi, Progress in Mathematics, 2, Birkhäuser, 1979.

[53] Quillen, D., "Formal properties of overdetermined systems of linear partial differential equations," Harvard PhD thesis, 1964.

[54] Ramis, J.-P., Géométrie analytique et géométrie algébrique (variations sur le thème "GAGA"), in "Séminaire Pierre Lelong-Henri Skoda (Analyse), Année 1976/77," Lecture Notes in Math., 694, Springer 1978, pp. 228-289.

[55] Rietsch, K., An introduction to perverse sheaves, in "Representations of finite dimensional algebras and related topics in Lie theory and geometry," Fields Inst. Commun., 40, Amer. Math. Soc., 2004, pp. 391-429,.

[56] Roos, J.-E., "Détermination de la dimension homologique globale des algébres de Weyl," C. R. Acad. Sci. Paris Sér. A-B 274, (1972), A23-A26.

[57] Sabbah, C. Quelques remarques sur la géométrie des espaces conormaux, Astérisque 130 (1985), 161-192. 
[58] Sato, M., Kawai, T., Kashiwara, M., Microfunctions and pseudo-differential equations, in "Hyperfunctions and pseudo-differential equations," (Proc. Conf., Katata, 1971; dedicated to the memory of André Martineau), Lecture Notes in Math., 287, Springer, 1973, pp. 265-529.

[59] Schapira, P., "Microdifferential systems in the complex domain," Grundlehren der Mathematischen Wissenschaften 269 Springer-Verlag, 1985.

[60] Schapira, P., Letter to S. Kleiman, 8 March 1989.

[61] Schapira, P., Commentary on Kashiwara's contributions to the relevant $\mathcal{D}$-module theory, delivered to SLK by V. Guillemin, summer 1989.

[62] Schapira, P., Mikio Sato, a visionary of mathematics, transl. of Mikio Sato, un visionnaire des mathématiques, Gaz. Math. 97 (2003), 23-28; available at http://www.math.jussieu. $\mathrm{fr} /$ schapira/articlesdiv, to appear in the February 2007 Notices of the AMS.

[63] Schürmann, J., "Topology of singular spaces and constructible sheaves," Mathematics Institute of the Polish Academy of Sciences, Mathematical Monographs (New Series) 63 Birkhäuser Verlag, 2003.

[64] Schürmann, J. A general intersection formula for Lagrangian cycles, Compos. Math. 140, no. 4, (2004), 1037-1052.

[65] Schneiders, J.-P., An introduction to $\mathcal{D}$-modules, in "Algebraic Analysis Meeting (Liège , 1993)," Bull. Soc. Roy. Sci. Liège 63 (1994), no. 3-4, 223-295.

[66] Siegel, P., Witt spaces: A geometric cycle theory for KO-homology at odd primes, Amer. J. Math. 105 (1983), 1067-1105.

Steven L. Kleiman

Room 2-278, M.I.T.

Cambridge, MA 02139, U.S.A.

E-mail: kleiman@math.mit.edu 